\title{
Neural Timing Deficits Prevalent in Developmental Disorders, Aging, and Concussions Remediated Rapidly By Movement Discrimination Exercises
}

\author{
Teri Lawton ${ }^{1}$, John Shelley-Tremblay ${ }^{2 *}$ and Ming-Xiong Huang ${ }^{3,}$ \\ 1 Cognitive Neuroscience, Perception Dynamics Institute, PO Box 231305, Encinitas, CA 92023-1305; path- \\ toreading1@gmail.com \\ 2 Professor and Chair, Department of Psychology, University of South Alabama, Mobile, AL 36688; jstrem- \\ blay@usouthalabama.edu \\ 3 Professor \& Co-Director of MEG, Radiology Imaging Laboratory, Department of Radiology, University of \\ California, San Diego, 3510 Dunhill Street, San Diego, CA 92121-0852; mxhuang@ucsd.edu. \\ * Correspondence: jstremblay@usouthalabama.edu; Tel.: (1 (251) 460-6371)
}

\begin{abstract}
Background: Substantial evidence that neural timing deficits are prevalent in developmental disorders, aging, and concussions resulting from a mild Traumatic Brain Injury (mTBI) is presented. We show that if timing deficits are remediated using low-level movement discrimination training, then high-level cognitive skills, including reading, attention, processing speed, and working memory improve substantially. (2) Methods: Two case studies are presented using MEG source imaging on an adult dyslexic, and a healthy older adult observed before and after training on movement discrimination two times/week for 8 weeks for adult dyslexic. (3) Results: We found improvements in reading, attention, processing speed, and working memory on neuropsychological tests. Substantial MEG signal increases in visual Motion Networks (V1, V3, MT, MST), Attention Networks (ACC, dlPFC, vlPFC and precuneous/ PCC areas) and Memory Networks (dlPFC). (4) Conclusions: Improving neural timing deficits before cognitive exercises to improve specific cognitive skills provides a rapid and effective method to improve cognitive skills. Improving the timing and sensitivity of low-level dorsal pathways, improving feedforward and feedback pathways, is essential to improve high-level cognitive skills. This adaptive training with substantial feedback shows cognitive transfer to tasks not trained on, significantly improving a person's quality of life rapidly and effectively.
\end{abstract}

Keywords: Timing Deficits; Magnocellular Deficits; Remediating Cognitive Skills; Cortical Plasticity; Reading, Attention, Memory, and Executive Control Networks; Perceptual Learning

\section{Introduction}

For purposeful and useful interactions with the external world, the brain needs to integrate information processed in different parts of the nervous system, so that it can efficiently process sensory inputs, often from more than one modality, stored memories, emotional aspects of the situation, and executive and motor programs needed for the chosen response. This requires the operation of many brain areas communicating with each other to orchestrate and integrate the activity of the different cortical areas that are involved in a particular function by boosting the synchronized oscillations that occur between these areas. The neural timing deficits that are found in a wide range of brain disorders, affecting these synchronized oscillations are not well understood. We will describe the abundant evidence showing that neural timing deficits are prevalent in developmental disorders, like dyslexia where reading is difficult, normal aging, and concussions resulting from mild Traumatic Brain Injuries (mTBI). We will also describe a novel, break-through technique, called PATH to Reading/Insight neurotraining, that has been 
found to remediate these neural timing deficits, significantly improving cognitive abilities, including reading, processing speed, understanding, attention, and both auditory and visual working memory, so that a person's quality of life improves rapidly. These methods require a paradigm shift based on the neuroscience underlying the brain pathways used for detecting and discriminating events to see what is happening around us. By increasing the timing and sensitivity of the dorsal visual stream, used to locate the position of objects, cognitive abilities were found to increase significantly [1-6].

The movement direction-discrimination intervention (PATH neurotraining) is believed to improve the precision of timing of visual events and thus accelerate reading progress $[2,3,7,8]$. It achieves this by improving the function of the dorsal stream, boosting magnocellular relative to parvocellular activity, thereby improving inhibitory and excitatory circuits, based on the data from neural plasticity [9]. This theory is based on the idea that the synchronous firing of neurons is what controls communication between different areas in the brain [9]. If neurons in one area are "sluggish" with respect to neurons in another area, then they will be unable to synchronize properly. In this model, processing speed will be slowed down, and therefore communication, and hence learning, will be compromised. Experience refines the output of cortical circuits by introducing patterned activity that fine-tunes the strength of neuronal connections within and among cortical columns [9]. Neuroplasticity is fundamental for the brain's neural circuits to be malleable throughout a person's lifespan.

By extensive training on movement-discrimination, we found that we are improving the attention, executive control, and reading networks [4,5]. Visually-based movement discrimination exercises in both normal participants [1,6,7,10-13] and dyslexics [1$3,6,7,13,14]$ have demonstrated neuroplasticity in the domain of processing speed using massed practice. These studies found that the more movement discrimination was practiced, the more contrast sensitivity for movement-discrimination, reading, attention, and memory skills improved, with gains in speed, accuracy, comprehension, attention, and working memory being measured using age-appropriate standardized tests for these cognitive skills. Since improving the brain's timing improves cognitive skills, this indicates that timing is a key factor preventing normal cognitive function in dyslexia, aging, and mTBI. Not only was PATH training more effective, but it also required less than half the training time used by other reading and cognitive interventions.

We provide additional evidence showing that reading, attention, and memory problems involve neural timing issues caused by deficits in motion cells in the visual system's dorsal stream. These can be detected in the retina, lateral geniculate nucleus (LGN), visual cortical areas V1, V3, which are the input to the visual motion areas, the Middle Temporal (MT) and Medial Superior Temporal (MST) Cortex. These deficits in the dorsal stream affect feedforward and feedback pathways between visual, parietal and frontal areas.

\subsection{The visual system includes a fast dorsal stream providing input to attention networks}

The visual system has been hypothesized to exploit the dichotomy of a fast magnocellular channel (dorsal visual stream) and slower parvocellular channels (ventral visual stream) for the purpose of selective attention $[15,16]$. Receiving predominantly magnocellular input [17-19], the major dorsal stream pathway, specialized for processing the location and movement of objects in space [17,20,21], projects from the primary visual cortex, V1, through visual area MT (middle temporal cortex) to the medial superior temporal area (MST) [22], as well as projecting to V3, V3A and V6 [23]. Then it projects into the intraparietal sulcus of the posterior parietal cortex (PPC), a selective spatial attention area [24] that is also used to analyze event timing [25]. The PPC provides the input to the dorsal lateral PreFrontal Cortex (dlPFC) where working memory is encoded. The PPC and dlPFC being the predominant cortical areas, the hubs, that are involved in the Executive Control Network [26]. Executive control is a strong predictor for various life outcomes, such as academic achievement, since executive function is directly associated with math ability as well as with reading, writing, and language comprehension [27]. PATH neurotraining was one of only ten interventions found to increase executive function and brain 
fitness for students by Brain Futures [28]; brain fitness: neuroplasticity reveals that neurocircuitry in the brain is highly malleable, continuing to grow and change for the duration of our lives.

The ventral stream, in contrast to the dorsal stream, receives both magnocellular and parvocellular inputs as it projects from V1 through area V4 and on to the infero-temporal (IT) cortex, an area specialized for extracting the details related to an object's color and shape $[17,20,21]$. The faster transmission time of the magnocellular neurons projecting predominantly to the dorsal stream is gated via attentional feedback to the striate cortex [16], which can then be used by parvocellular neurons in the ventral stream as a starting point for deciphering the individual letters in a word [2,3,15,16,29-31]. Moreover, feedback in the dorsal stream from MT to V1 improves figure/ground discrimination [32], a task used when reading to distinguish the letters in the word being fixated from the surrounding text. Furthermore, feedback from MT has its strongest effects for low salience stimuli [32], such as low contrast patterns having less than $10 \%$ contrast, i.e. those patterns that maximally activate magnocellular neurons [33,34].

The dorsal visual stream provides the input to the attention networks [15] which are composed of two Attention Networks: 1) Exogenous Attention, called the Salience Network and 2) Endogenous Attention, called the Executive Control Network [26]. The Salience Network operates using dynamic switching, having hubs in the anterior insula (AI) and the anterior cingulate cortex (ACC), by activating the Central Executive Control Network and at the same time suppressing the Default Mode Network [26]. The two major hubs of the Executive Control Network used for cognitively challenging tasks are the PPC and the dlPFC [26]. The control of spatial attention in early visual cortex is likely to be directed by regions of the PPC and dlPFC [35]. Neural timing deficits will prevent the correct functioning of these two attention networks, especially when caused by slow magnocells that are found at all levels of the dyslexic's visual dorsal stream, as described in the Discussion.

When reading, it has been proposed that the PPC uses the spatial information of the location and overall shape and form of a word that is received through the rapid magnocellular pathway to gate the information that is going into the temporal stream [16]. The information is gated via attentional feedback to the striate cortex and to other regions in the occipito-temporal cortex [15,16,36-38] most likely done by top-down feedback which uses synchronized neuronal oscillations at the lower end of the gamma frequency range [15]. Since top-down attentional signals from PPC to Frontal Eye Fields (FEF) [39] and from PPC to MT [40] have been identified, as well as top-down signals from the PPC to MT and then on to V1[16,41], modulation of gamma oscillations during reading might arise from the 'back-end' of the dorsal stream network. Archer, Pammer, and Vidyasagar [42] hypothesize that this occurs in the PPC where increased gamma activity is shown to be linked to visual attention and planned saccadic eye movements. This in turn is used by the parvocellular neurons in the ventral stream, using coupled alpha-gamma oscillations regulated by the pulvinar for sequential processing [43], as a starting point for deciphering the individual letters $[15,16]$. In fact, the visual word form area (VWFA) in the ventral stream, where the visual shapes of words are analyzed in detail [44] receive significant magnocellular input from the dorsal stream to direct the VWFA's attention to which word it should analyze next $[15,45]$. It is likely that the dyslexic reader's deficit in attentional focus $[16,29,46,47]$ is a consequence of sluggish magnocellular neurons preventing the linked parvocellular neurons from being able to isolate and sequentially process the relevant information that is needed for reading [16,29,48], and not from an information overload as was proposed previously [49].

Each cycle of gamma oscillation focuses an attentional spotlight on the primary visual cortical representation of just one or two letters, in order to recognize those letters in the right sequence to concatenate them into words [15]. The timing, period, envelope, amplitude, and phase of the synchronized oscillations that is modulating the incoming signals to the striate cortex have a profound influence on the accuracy and the speed of reading [15]. The speed determined by the gamma frequency oscillation is the essential rate- 
limiting step in dyslexia [15]. It is proposed that impaired theta and gamma frequency oscillations in the visual domain hinder effective visual temporal sampling and parsing of text [42]. Cross-frequency theta-gamma coupling enables sensory areas of the brain which capture language stimuli to communicate rapidly with higher-order brain areas for real-time processing of language input [42,50]. Impaired theta-gamma oscillations also underlie the impaired coding of spoken syllables and phonemes, which is essential for phonological processing [51]. Sequential processing uses the functional anatomy of the claustral connections of items being processed serially, such that cross-frequency coupling between low frequency (theta) signals from the claustrum and higher frequency oscillations in the cortical areas is an efficient means of the claustrum modulating neural activity across multiple brain regions in synchrony [52]. In addition to the claustral connections mediating this theta-gamma cross frequency coupling, coupled alpha-gamma oscillations regulated by the pulvinar are used for sequential processing [43] to read words, for example. Both claustral connections [52] and the pulvinar complex [43] regulate synchronous information transmission between cortical areas based on attentional demands. Cross-frequency coupling is being recognized as an efficient means of communication between multiple cortical areas that is likely to play a crucial role in mediating working memory and in enabling learning [53,54].

1.2 Improving visual dorsal stream function remediates reading fluency, attention span, and memory retention

The motion (magno) cells in dyslexics are responding up to $40 \mathrm{msec}$ slower than they should be $[55,56]$. Therefore, the motion (dorsal) pathway and the pattern (ventral) pathways are not in sync and working together optimally, since the dorsal brain pathways are slowed down. This slowdown causes problems with paying attention, multitasking, sequential processing and remembering easily [1-6,57], as described in this short movie: https://youtu.be/LDdhuhPeXNI. Sluggish motion cells make it difficult to locate the beginning and end or identify the order of letters in a word, causing confusion, mis-sequencing, and hence slow reading. Slow neural pathways cause the brain to misdirect visual attention, confuse what the eye sees, and reduce the ability to remember the visual forms of words.

The sluggish magnocellular neurons in dyslexics not only result in attention deficits, causing an impairment in the low gamma frequencies that reduce feedback to visual cortical areas [15], but it also disrupts processing in the Lateral IntraParietal (LIP) area. This area keeps a salience map for the control of saccadic eye movements and visual attention [58] and FEF, either within a fixation, between a fixation sequence, or both [16,59-61]. Its disruption causes very slow reading speeds. Moreover, finding that left-right movementdiscrimination training improved not only reading fluency, but also selective and sustained attention, and working memory when done before reading or other cognitive exercises [3-6] indicates that movement-discrimination training (PATH to Reading/Insight: https://pathtoreading.com) helps develop the attention and executive control networks. These results provide more evidence showing that slow visual motion processing is a fundamental cause of reading and attention problems in dyslexia and other cognitive slowdowns, like those caused by a concussion [5]. By improving the attention network's functioning, movement-discrimination training provides a wider window of attention, so that more objects are perceived in their correct location in a single glance [62]. Movement direction-discrimination training also improves the ability to detect the synchronicity of multiple objects in space and see their trajectories over time, most likely by increasing the ease of magno-parvo integration, thereby facilitating figure/ground discrimination within a wider window of focused attention [6]. Moreover, there is evidence that improvements in the reading speed of dyslexics after movement-discrimination training are sustained over time [2], whereas improvements in word reading found following auditory interventions to improve phonological processing degrade over time, two years later showing no difference in word reading skills when compared to controls who did not complete the 
auditory intervention [63]. Moreover, improvement following visual movement-discrimination training is significantly faster and more effective than auditory movement-discrimination training (FastForWord) as shown in a randomized clinical trial [3].

Improving cognitive function by training left-right movement discrimination relative to a background is a novel method [4,7] that was found to be both rapid and effective in improving cognitive skills in dyslexics, as well as those with mTBI [5] and older adults [62]. Only when low-level visual timing deficits, in slow magnocells are remediated are the improvements in high-level cognitive functions, such as reading fluency (speed and comprehension), attention, processing speed, and working memory improved quickly, with improvements that are sustained over time [2]. PATH neurotraining is the first visually-based intervention that was found to improve both low-level movement discrimination in the dorsal stream and high-level cognitive functioning, both behaviorally and using MEG brain imaging, improving the attention and the executive control networks in dyslexics [4] and mTBI [5]. Since movement-discrimination (PATH) neurotraining is so rapid and effective, it offers a new approach that represents a paradigm shift in the treatment of dyslexia, one that is based on improving visual timing instead of improving phonological timing, as used by FastForWord. When reading, students who allocate all their resources to identify the letters in the word, instead of allocating their resources to interpret a sentence, understand its meaning, and integrate the information into their existing knowledge need movement figure/ground discrimination training to remediate their visual timing deficits.

Clearly, there is a need to provide this novel treatment not only for individuals who have sustained a concussion or traumatic brain injury, for which there are no proven solutions to improve working memory in mTBI patients [64], but also for people with learning problems caused by dyslexia, as well as speech and language problems, attention deficits, autism, and schizophrenia. As indicated by recent scientific studies, magnocellular deficits are a major factor in these diseases [65,66].

PATH neurotraining measures contrast sensitivity for direction discrimination of left-right movement, since the cells in the motion area MT are optimally tuned to the direction of moving patterns [67]. The direction of movement is tested relative to stationary, textured background patterns that enable the lowest movement discrimination thresholds to be measured [10-12]. Moreover, these moving patterns consist of dim achromatic stripes, since these are the patterns optimal for activating magnocellular (magno) neurons. In fact, once the contrast (difference in luminance between dark and light bars) exceeds $10 \%$, the magnocells saturate and no longer convey new information to higher processing levels [33]. Normally the motion cells signal not only the beginning and end of each word but also shifts of attention or eye movements from letter to letter, enabling the highresolution pattern cells (called parvocellular neurons) to fill in the details. However, when the motion cells are not fast enough, this delay causes many problems.

PATH training in movement figure/ground discrimination improved not only magnocellular function, attention, processing speed, and memory, but also improved magnoparvo integration, figure/ground discrimination, and is hypothesized to increase coupled theta-gamma activity for the test patterns moving at 6.7 and $8 \mathrm{~Hz}$ and coupled alpha/gamma activity for test patterns moving at 10 and $13.3 \mathrm{~Hz}$. After doing PATH neurotraining, test patterns moving at 10 and $13.3 \mathrm{~Hz}$ were the patterns that improved in contrast sensitivity the most $[2,3,6]$. They were not discriminated until direction-discrimination for more slowly moving patterns were learned. Both coupled alpha-gamma and theta-gamma oscillations are key for efficient reading skills, both being trained by PATH neurotraining, and validated by improving reading speeds 11-fold after only 12 weeks of training for 20 minutes twice a week [2].

To verify that these improved cognitive skills following PATH training are also found in the dorsal visual stream (V1, V3, MT, and MST) as well as in the attention networks (ACC, precuneus/PCC, PPC, dlPFC, and vlPFC of a dyslexic, MagnetoEncephaloGraphy (MEG) brain source imaging, providing a biomarker, was conducted to determine the brain areas that increase in function for an adult (29 years-old) dyslexic following 
PATH training [4], with data never before published. This biomarker for dyslexia shows for the first time that PATH training improves the function of the motion area: MT in the first $300 \mathrm{msec}$, improving the sensitivity and neural timing of magnocells in the dorsal stream. Improvements in cognitive skills were also measured for an older adult (71 yearsold) with a battery of cognitive skills standardized tests, for the first time. These results validate the reported improvements in cognitive skills found previously [62] using robust neuropsychological tests. Since coupled alpha-gamma activity is reduced in older adults with mild cognitive impairments resulting from memory loss [68], the improvements found in this person's working memory provides more evidence PATH training improves both coupled alpha-gamma and theta-gamma activity. For both people, improvements in cognitive skills were measured using a battery of standardized tests before and after 8 weeks of PATH neurotraining twice a week for 15-20 minutes, providing more rigorous evidence than available previously.

\section{Materials and Methods}

\subsection{Participants}

A 29 years-old dyslexic man answered an ad to improve cognitive skills that was posted by UCSD. He had been finding his quality of life was limited by his dyslexia and was interested in any methods to improve his cognitive skills. A healthy 71 years-old woman, having a PhD in physics, referred by a professor at UCSD, enrolled in this study, since she wanted to improve her ability to remember. Lately, she found her memory was not as reliable, increasingly reducing her quality of life, causing frustration in daily activities, especially when driving during dawn or dusk.

Both participants signed informed consent forms before they participated in this study. The dyslexic subject's consent form was approved by UCSD Institutional Review Board, and the older adult's consent form was approved by Solutions IRB, a full service private Institutional Review Board registered with OHRP. The study was conducted in accordance with the Declaration of Helsinki, and the protocol was approved by each IRB.

\subsection{PATH Neurotraining}

PATH neurotraining uses dim grayscale patterns to retrain the brain's pathways. These patterns are designed to activate motion pathways (by using left-right movement) relative to the pattern pathways by using a stationary background that entrains motion discrimination (Lawton, 1985, 1989). Each pattern is presented for less than half a second, see Fig. 1 below. Only the contrast of the center stripes (the test frequency) in the fish shaped object that moves left or right relative to a stationary striped background is dimmed until the direction can no longer be seen. The low contrast $(0.1-5 \%)$ test frequency was set to either $0.25,0.5,1$ or $2 \mathrm{cyc} / \mathrm{deg}$, being an octave apart, since spatial frequency neural channels are sensitive to one octave of different spatial frequencies [69]. Based on physiological data, the lowest spatial frequency channel is $1 \mathrm{cyc} / \mathrm{deg}$ [69] requiring summation across neural channels to discriminate the direction the $0.25 \mathrm{cyc} / \mathrm{deg}$ test frequency moved. The five stationary vertical background gratings for each test frequency consisted of single and multiple spatial frequencies at increasing levels of complexity. The background gratings whose fundamental frequency is equal to the test frequency or \pm 1 or 2 octaves from the test frequency were chosen to bracket the test frequency. These patterns were designed to activate adjacent spatial frequency channels in V1 and MT $[70,71]$. There are 16 levels of complexity, each increasing in difficulty slowly, from slow theta movement $(6.7,8 \mathrm{~Hz})$ to faster alpha movement $(10,13.3 \mathrm{~Hz})$ for both one (Motion Program) and two (MotionMemory Program) directions of left-right movement. The feedback to the user makes this a game that motivates the user to continue to improve. 


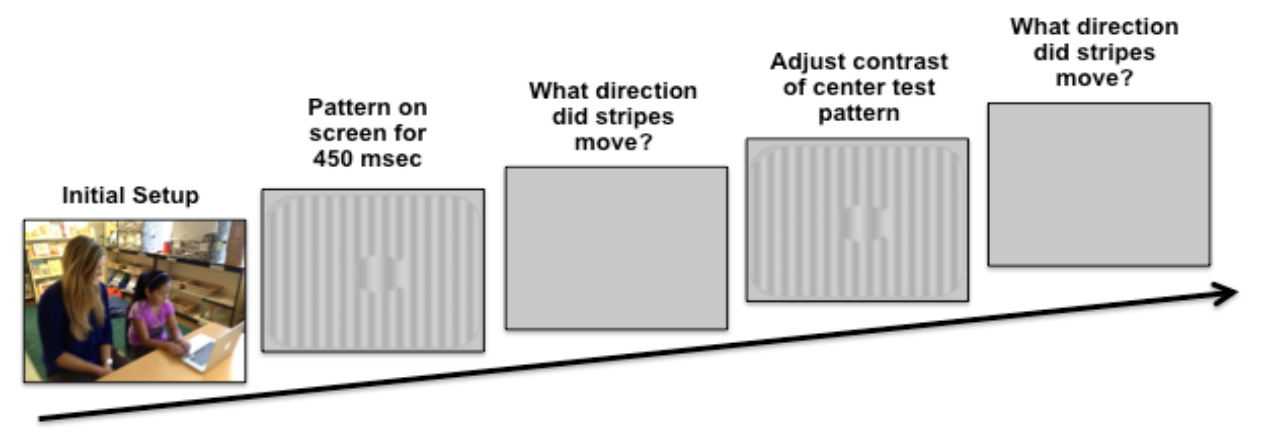

Figure 1. Schematic of Stimulus Presentation for PATH to Reading /Insight intervention. Pattern flashes on screen (shown above) while center stripes move left or right. Screen goes blank, waits for left or right arrow key to be pushed. If incorrect, short tone sounds. Pattern with same or different contrast flashes on screen while center stripes move left or right. Screen goes blank, waits for left or right arrow key to be pushed. This sequence of patterns is presented continuously until the contrast threshold for this pattern is measured. Then the next pattern combination is presented to measure next contrast threshold until all 20 PATH neurotraining patterns were presented, and the program says 'Thank You', presents a star for each level of complexity completed, and quits.

The subject was seated an arm's length $(57 \mathrm{~cm})$ from the computer screen. At the start of a session, both the test and background gratings were set to $5 \%$ contrast to ensure that the contrast of the test pattern was in the middle of the magnocellular contrast range [33]. The test pattern subtends 4 deg visual angle surrounded by a background that subtends $16 \mathrm{deg}$ visual angle. The mean luminance of the patterns was $125 \mathrm{~cd} / \mathrm{m} 2$ calibrated using a Spectra Pritchard 1980A Photometer. Each time the direction the fish stripes moved was identified correctly, the contrast of the test grating was lowered until the first incorrect response. Following the first incorrect response, a double-staircase procedure was used to estimate the direction-discrimination contrast threshold, which allowed measuring the contrast sensitivity, defined as the reciprocal of the contrast threshold times 100. This staircase procedure estimates the contrast needed for $79 \%$ correct responses, providing the most sensitive, repeatable measurements of contrast sensitivity [72]. This training was adaptive on a trial-by-trial basis in response to the subject's performance, such that lower contrasts were presented in response to good performance to improve sensitivity to motion at each of four durations and as the complexity of the background increased. Moreover, the training incorporated cycles of feedback and reward at multiple levels, ranging from positive and negative feedback on a trial-by-trial level, as well as cumulative block and session feedback. It has been found that feedback greatly accelerates learning [73,74]. A full training cycle of the direction-discrimination task required 20 threshold determinations (i.e. one for each of the four test spatial frequencies paired with each of the five background spatial frequencies).

The complexity level increased the: 1) number of sinewave components in the background from one to three, providing more structured background frame of reference enabling lower contrast thresholds [11,12], 2) background contrast from $5 \%$ to $10 \%$ to $20 \%$, and 3) pattern's speed of movement after every 4 complexity levels, increasing from 6.7 $\mathrm{Hz}$ to $8 \mathrm{~Hz}$ to $10 \mathrm{~Hz}$ to $13.3 \mathrm{~Hz}$, so that the student was challenged as the training progressed. The background contrast was increased to $20 \%$ contrast to provide a background that increased parvocellular activity, since magnocellular neurons saturate at $10 \%$ contrast [33]. The $20 \%$ contrast background required students to analyze information from magnocellular activity relative to increased parvocellular activity, making the task more challenging. The order of presentation for each complexity level was chosen to gradually increase the difficulty of the task [2]. Therefore, as the complexity level increased, the contrast sensitivity should be lower initially. Once all 16 complexity levels of the Motion program were completed, the subject progressed onto the next program, 
the MotionMemory program. Instead of discriminating the direction one pattern moved by pushing the left or right arrow key as in the Motion program, MotionMemory requires signaling the direction that two separate patterns moved, one after the other, by pushing one of four arrow keys. Each threshold in both the Motion and MotionMemory programs required 20-40 trials to complete. A score was given to make the training more game-like. The lower the contrast threshold, the higher was the score. After learning how to do this task, each subject typically took about 15-20 minutes to complete one training cycle, consisting of 20 contrast thresholds. Motion direction-discrimination was trained for between 15-20 minutes, twice a week for 8 weeks.

This patented movement discrimination program [7,8] called PATH to Reading/Insight uses positive feedback that is provided in the form of catching more fish in a net resulting from meeting specific thresholds-, i.e. less than or equal to $1 \%$ contrast, and through a score that increases as performance improves. There is also immediate feedback in the form of a beep if the user identifies the direction of movement incorrectly, in addition to other verbal feedback when needed. Simply interacting with a game-like training program for 8 weeks on a computer for 20 minutes twice a week, is all that was involved.

2.2.1 Fidelity of Implementation: All contrast threshold data with date and time stamps was stored in individual and summary files, and collected automatically by the computer. Therefore, there was no means for tampering with the data collection. Data in summary files showed each subject's contrast thresholds, how long it took to complete each threshold. At the end of each training cycle, the subject earned a star for each level of complexity completed to let them follow their training progress more easily.

\subsection{MEG Source Imaging}

MEG responses evoked by a $2.5 \%$ contrast $1 \mathrm{cyc} / \mathrm{deg}$ sinewave grating relative to a $5 \%$ contrast $1 \mathrm{cyc} / \mathrm{deg}$ background moving left or right at $10 \mathrm{~Hz}$ was collected using the VectorViewä whole-head MEG system (Elekta-Neuromag, Helsinki, Finland) with 306 MEG channels. This task was used, since sensitivity to a $1 \mathrm{cyc} / \mathrm{deg}$ moving pattern was based on output from single spatial frequency channels used for movement discrimination. Meta-analyses indicate that the working memory network consists of six reliably activated cortical regions [75,76]: (1) bilateral rostral prefrontal cortex (rPFC) including frontal pole (FP, BA 10), ventromedial prefrontal cortex vmPFC, and orbitofrontal cortex (OFC, BA 11); (2) bilateral dlPFC (BA 9, 46); and (3) bilateral ventrolateral prefrontal cortex (vlPFC) or frontal operculum (BA 45,47); (4) bilateral medial posterior parietal cortex (PPC), including the precuneus, and the inferior parietal lobules (approximate BA7,40); (5) bilateral premotor cortex (BA 6, 8); and (6) dorsal cingulate/medial premotor cortex, including supplementary motor area (SMA; BA 32,6). The cerebellum is also consistently activated during WM.

The dyslexic subject underwent MEG recordings while performing the task above. The task entails on-line monitoring, updating, and manipulation of remembered information. During the task, the subject was required to monitor the direction of movement. A fixation cross was presented during the $3000 \mathrm{~ms}$ interstimulus interval. The subject was instructed to push a right button if the test pattern moved right relative to the background and push a left button if the pattern moved left relative to the background. About 50 trials per load condition were collected for this subject. Performance was recorded using an MEG-compatible response pad, in which index finger blocks-and-unblocks a laser-beam.

The dyslexic subject was seated in an upright position inside a multi-layer magnetically-shielded room (IMEDCO-AG) at the UCSD MEG Center. Two MEG exams were performed for this subject who did not have metals objects in his brains, one before and another after the movement-discrimination intervention. MEG data were sampled at 1000 $\mathrm{Hz}$ and were run through a high-pass filter with a $0.1 \mathrm{~Hz}$ cut-off, and a low-pass filter with a $330 \mathrm{~Hz}$ cut-off. Eye blinks and eye movements were monitored using two pairs of bipolar electrodes with one pair placed above and below the left eye, and the other pair placed on the two temples. Heart signals were monitored with another pair of bipolar 
electrodes. Precautions were taken to ensure head stability; foam wedges were inserted between the subject's head and the inside of the unit, and a Velcro strap was placed under the subject's chin and anchored in superior and posterior axes. Head movement across different sessions was about 2-3 $\mathrm{mm}$ on average.

MEG sensor waveforms in raw (un-averaged) format were first run through MaxFilter, also known as signal space separation [77-79], to remove external interferences (e.g., magnetic artifacts due to metal objects, strong cardiac signals, environment noises, etc.). Next, residual artifacts near the sensor array due to eye movements and residual cardiac signals were removed via Independent Component Analysis using Fast-ICA (http://research.ics.aalto.fi/ica/fastica/) [80,81]. The waveforms associated with top independent components (ICs) were examined by an experienced MEG data analyst, along with ECG and EOG signals. ICs associated with eye blinks, eye movements, heartbeats, and other artifacts were removed.

Following the pre-processing step, MEG sensor-waveform datasets were run through band-pass filters for beta band $(15-30 \mathrm{~Hz})$. Each data set was then divided into trials, each with 2.5-second duration (-500 ms to $1500 \mathrm{~ms}$ with respect to the stimulus onset). In the present study, we focused on the trials associated with target stimuli.

Different from the conventional MEG approach in which sensor waveforms are averaged with respect to the onset of the stimuli, the sensor covariance matrices for individual trials were calculated. Then a total sensor-waveform covariance matrix of the target condition was calculated by averaging across the covariance matrices from individual trials for the target stimuli. Then the covariance matrices across trials were averaged. Using the total covariance matrix, voxel-wise MEG source magnitude images that cover the whole brain were obtained for the dyslexic subject, and each frequency band, following the Fast-VESTAL procedure [82,83], measuring time-locked signals during a movement discrimination task to evaluate improvements in brain function. An objective pre-whitening method was applied to remove correlated environmental noise and objectively select the dominant eigen-modes of sensor-waveform covariance matrix [82].

Voxel-wise whole brain MEG source magnitude images obtained from Fast-VESTAL were first spatially co-registered to the MNI-152 [84] brain-atlas template using a linear affine transformation program, FLIRT, in the FSL software package $[85,86]$. Then in MNI152 space, the MEG source magnitude images were spatially smoothed using a Gaussian kernel with $5 \mathrm{~mm}$ full width half maximum (FWHM), followed by a logarithmic transformation using FSL. Next, in MNI-152 coordinates, voxel-wise paired-sample t-test statistical analysis was performed to detect differences in brain activation during the MEG movement discrimination task before and after the PATH neurotraining intervention. Cluster analysis with threshold of corrected $p=0.01$ was used to correct the family-wise error across voxels.

2.4 Behavioral improvements in cognitive skills were measured using a battery of standardized and reading skill tests

The following standardized tests of reading, attention, and working memory skills were administered by trained staff at the beginning and end of 8 weeks of PATH neurotraining twice a week for 20 minutes. These tests provide a standardized measure of the improvements in high- level cognitive functions after PATH neurotraining. They were chosen because these tests are the "Gold Standard" tests for fast and accurate measurements of fluency-based reading, attention, processing speed, and working memory (both visual and auditory) skills. The tests of visual and cognitive skills that were completed are:

1. Movement Discrimination Diagnosis program for dyslexic subject, consisting of first session of PATH neurotraining to determine whether the subject had difficulty doing left-right movement discrimination, being characterized by 6 levels of movementdiscrimination: 1-above normal, 2-normal, 3-borderline movement discrimination deficits, 4-mild movement discrimination deficits, 5-moderate movement discrimination deficits, and 6-markedly below normal movement discrimination deficits. 
2. Adult Dyslexia Test for Older Adult measures how many words read correctly in 2 seconds or less at each grade level up to post graduate after doctorate. Words that are pronounced correctly in 2 second or less are then spelled. Words not able to be pronounced correctly are spelled phonetically. Based on the number of correctly spelled words at each grade level compared to actual grade level, proficiency is evaluated as above normal, normal, borderline, mildly below normal, moderately below normal and markedly below normal.

3. Wide Range Achievement Test (WRAT) for Older Adult measures number of words read correctly (Reading), spelled correctly (Spelling), and number of math problems solved correctly (Math). Based on a person's age, a standardized percentile score was assigned.

4. Computer-Based Reading Speed test, not limited by the person's rate of speaking, was completed by determining the speed needed to read 6 words of text on the screen from an interesting story (Cheaper by the Dozen) at increasing speeds to measure 2 reading rate thresholds ( $79 \%$ correct) using a double-staircase procedure, after being trained by watching a 4-minute movie.

5. Attention tests for Dyslexic measured using Integrated Visual and Auditory Continuous Performance Plus (IVA+Plus) Tests to measure attentional focus, sustained attention, or inattention, hyperactivity, and impulsivity were converted to standardized percentile scores. (15 min).

6. Attention tests for older adult measured using Delis-Kaplan Executive Function System (DKEFS) Color-Word Interference test, where subject had to say the printed color of words that denoted a different color (Stroop Attention test), and Attention Switching (Cognitive Flexibility), switching between the color of the word and what the word says when surrounded by a rectangular box. Standardized scores were converted to standardized percentile scores.

7. Wechsler Adult Intelligence Scale (WAIS)-4 Processing Speed required two subtests: the WAIS Symbol Search subtest which required subjects to scan a target group and search a group of symbols, indicating whether one of the target symbols appeared in the search group, and the WAIS Digit Symbol Coding subtest, where the subject filled in boxes below digits with symbols that were paired with them in a key at the top of the page. Both of these subtests were timed for two minutes each. The scaled scores from each subtest were combined to create an overall Processing Speed Index score, that was converted to a standardized percentile score.

8. WAIS-4 Working Memory Index is based on two subtests to measure Auditory Working Memory (AWM): 1) the Digit Span subtest, where the subject had to repeat a list of spoken numbers, requiring the subject to remember subsequently more numbers: in the correct order, backwards, and in numerical sequence on three different subtests, and 2) the Letter-Number Sequencing subtest which required sequencing subsequently more numbers and letters in the correct numerical and alphabetic sequence. Presentation of the numbers and letters were timed for one second each for these working memory tests. The standardized AWM score was converted to a standardized percentile score.

9. Visual Working Memory (VWM)) using the Test of Information Processing Skills (TIPS). The subject recalled a sequence of letters presented visually one at a time: right after seeing the entire sequence of letters, after counting 10 numbers in sequence, and after repeating a short sentence with animal names for the VWM task, and being asked to remember these animal names 3 minutes after completing the VWM task to measure Delayed Recall. Presentation of the letters were timed for two seconds each for this task. Standardized scores were converted to standardized percentile scores.

In addition to tests of cognitive skills, the dyslexic subject's visual skills: near point of convergence, and breaking and recovery points for near-point fixation disparity were 
measured to determine whether PATH neurotraining improved convergence insufficiency. All of these cognitive assessments, which were age-appropriate, took one hour to complete.

\section{Results}

Both MEG brain imaging, see Fig. 3, and behavioral tests, see Tables 1 and 2, found substantial improvements in the visual, attention, and executive control networks after 8 weeks of PATH neurotraining twice a week for 15-20 minutes.

\subsection{Dyslexic Adult Improved in Reading, Attention, Processing Speed, and Memory}
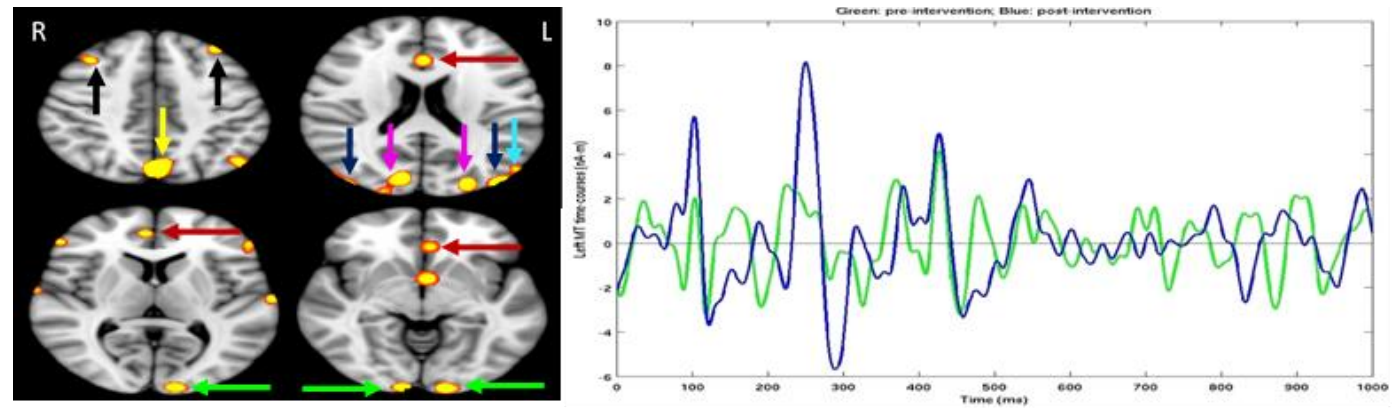

Figure 3. Significant MEG source magnitude (i.e., root-mean-square measure) signal increases (color scale for t-test: 3 - 3.5) in post- versus pre-intervention exams for an adult male dyslexic aged 29 years. Green arrows: V1; Blue arrows: MT; Magenta arrows: V3; Cyan arrow: MST; Red arrows: ACC; Yellow arrow: precuneus/PCC; Black arrows: dlPFC; Right Panel: MEG source timecourses from left MT area during post-intervention (Blue line) and pre-intervention (Green line) exams in the figure above.

Notice that the improvements in Fig. 3 happen in the first $300 \mathrm{msec}$, showing the training speeds up magnocellular activity in the motion area $\mathrm{MT}$, as seen in the right panel. PATH neurotraining improves the function of many related brain pathways, improving the visual, attention, and memory networks, as shown by MEG brain imaging conducted by Dr. Ming-Xiong Huang at UCSD after only 8 weeks of PATH training 2 times/week. The improvements in MT reveal improvements in P1 and N1 at durations between $80-150$ msec that result from alpha activations $(10-13.3 \mathrm{~Hz})$, and in P2 and N2 at durations between $240-300 \mathrm{msec}$ from theta activations (6.7 to $8 \mathrm{~Hz}$ ), improvements in N1 and $\mathrm{N} 2$ found for older adults following perceptual training on $10 \mathrm{~Hz}$ and $5 \mathrm{~Hz}$ moving patterns, respectively [87]. The visual P1 and N1 components reflect early sensory processing in visual cortices that are modulated by top-down attentional control [88]. The improved N1 and N2 components in Fig. 3, known to be enhanced by selective attention [89], were found after only 5 hours of perceptual training, whereas those from older adults in study of Mishra et al. [87] required twice as much training. Substantial MEG signal increases in visual Motion Networks (V1, V3, MT, MST), Attention Networks (ACC, dlPFC, vlPFC and precuneous/ PCC areas) and Memory Networks (dlPFC) were observed following training on direction-discrimination 2 times/week for 8 weeks for a 29 years adult dyslexic (first presented by Lawton \& Huang [4]). Notice that the left cortical areas V1 and MT showed more improvements than the right V1 and MT. Finding larger improvements on the left side is consistent with previous imaging studies showing that dyslexics have reduced activations on the left temporal, parietal, and fusiform (VWFA) regions [90]. These improvements in the left hemisphere visual, attention, and memory networks are also validated behaviorally.

Behavioral tests, shown in Table 1, found that not only did his ability to discriminate left-right movement improve from mildly below normal to above normal, his reading speed improved almost 3-fold, his attention skills improved remarkably, improving both focusing from $1 \%$ to $54 \%$, sustained attention from $10 \%$ to $82 \%$, and impulsivity from $18 \%$ to $62 \%$, attention improving an average of 22 -fold, his processing speed more than doubled, from $23 \%$ to $50 \%$, visual working memory improving 17 -fold, from $6 \%$ to $99 \%$, and 
delayed recall 25-fold. In addition, his visual skills improved substantially: his near point of convergence was reduced from $9 \mathrm{~cm}$ down to $3.5 \mathrm{~cm}$, and his near point fixation disparity breakpoint went from $29 \mathrm{~cm}$ to $18 \mathrm{~cm}$ and recovery went from $31 \mathrm{~cm}$ to $20 \mathrm{~cm}$. Therefore, these improvements show that PATH neurotraining reduces convergence insufficiency. In addition to these improvements in visual and cognitive skills, his quality of life also improved remarkably, doing activities that previously were too overwhelming to consider. He felt so secure that he decided to get married, and started a new business, an online app to help other dyslexics who need text magnified to be able to read online menus, for example. He also finished his Bachelors' Degree at College.

Table 1. Dyslexic Pre-Post Standardized Percentiles and Reading Scores

\begin{tabular}{|c|c|c|}
\hline Standardized Tests & Pre-Test & Post-Test \\
\hline Reading Speed & $154 \mathrm{wpm}$ & $437 \mathrm{wpm}$ \\
\hline IVA+ Focusing Attention & $1 \%$ & $54 \%$ \\
\hline IVA+ Sustained Attention & $10 \%$ & $82 \%$ \\
\hline IVA+ Impulsivity & $18 \%$ & $62 \%$ \\
\hline WAIS Processing Speed & $23 \%$ & $50 \%$ \\
\hline TIPS Visual Working Memory & $6 \%$ & $99 \%$ \\
\hline TIPS Delayed Recall & $1 \%$ & $25 \%$ \\
\hline
\end{tabular}

These improvements in cognitive skills found for dyslexics following PATH movement-discrimination training were not found by improving auditory timing using FastForWord nor linguistic-based training using Learning Upgrade [3], nor by computerbased repeated reading using Raz-Kids [6]. When compared to PATH neurotraining, repeated reading interventions do less to improve reading fluency, as supported by finding: 1) reading speeds improved only 2 -fold following repeated reading exercises [91], instead of from 3-fold to 11-fold, when reading aloud with a student following PATH neurotraining [2], and 2) improvements in comprehension using repeated reading interventions are much lower than found using PATH neurotraining. For example, Vadasy \& Sanders [91] found that repeated reading aloud improved comprehension $8 \%$, assessed by the Gray Oral Reading Test (GORT), whereas PATH neurotraining improved comprehension, when assessed by the GORT, 28\% for dyslexic students and 37\% for typically-developing students [6], even though each group trained for half as much time as done by Vadasy \& Sanders [91]. The improvements in cognitive skills following PATH training were also found in typically-developing children [6] who were in second and third grade (6 to 8 year olds), which is the age when the temporal lobe shows peak synaptogenesis [92]. PATH movement-discrimination training was also found to significantly improve reading fluency in typically-developing 6 to 8 year-old children previously [1,13,57]. Since timing impairments can be reduced following training using PATH movement-discrimination brain exercises $[3,6]$, these findings support the hypothesis that visual magnocellular pathways provide the gateway for attentive processing [15,16] and reading [66,93].

\subsection{Older Adult Improved in Reading, Attention, Processing Speed, and Working Memory}

The older adult's contrast sensitivity for movement discrimination when conducting PATH neurotraining for each test frequency, when averaged across the five background patterns, at each level of complexity is shown in Fig. 4. These contrast sensitivities are amongst the highest yet recorded, showing she was able to do this task easily, and age was not a limiting factor. The contrast sensitivities for the widest bars, $0.25 \mathrm{cyc} / \mathrm{deg}$, were much lower than the contrast sensitivities for the other test frequencies. These results support the hypothesis that information from several spatial-frequency neural channels must be combined to discriminate the direction of movement for a $0.25 \mathrm{cyc} / \mathrm{deg}$ test frequency, since $1 \mathrm{cyc} / \mathrm{deg}$ is the lowest spatial frequency channel [69]. This also explains why participants report that discriminating the direction of movement of $0.25 \mathrm{cyc} / \mathrm{deg}$ test 
frequency is the most difficult. The highest contrast sensitivities were found for the 2 cyc/deg test frequency. A similar pattern of contrast sensitivities for movement discrimination in older adults was found previously [62].

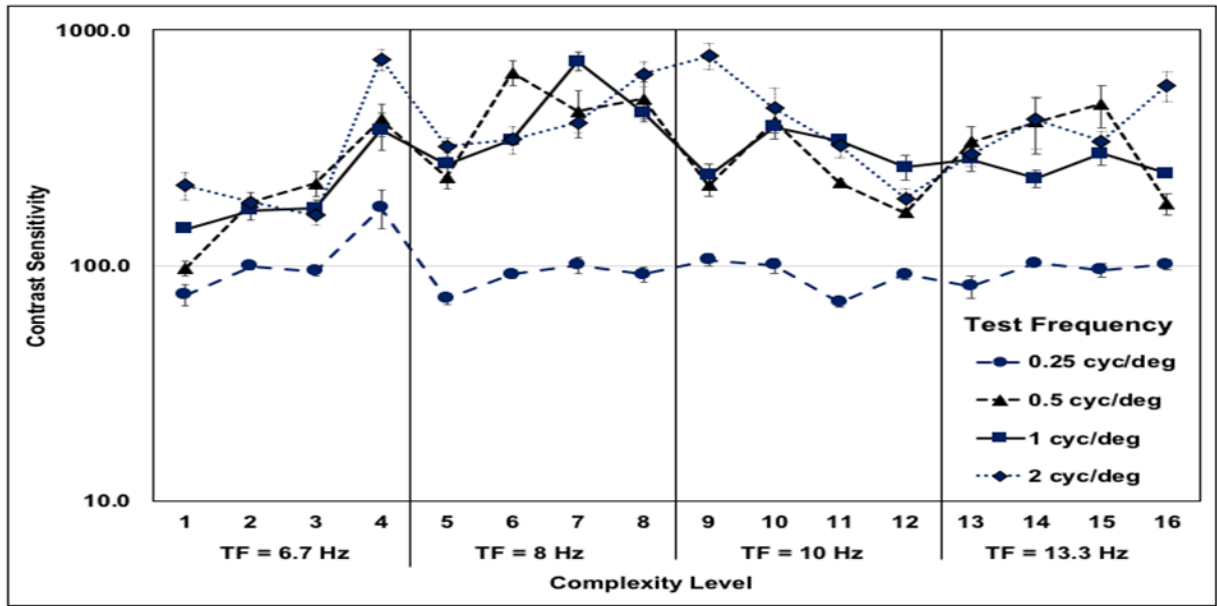

Figure 4. Older adult mean contrast sensitivity function for left-right movement discrimination at each complexity level, for each test frequency, when averaged across the five background patterns, each 4 complexity levels having a faster test frequency temporal frequency (TF). The first eight complexity levels training coupled gamma/theta oscillations and the second eight complexity levels training coupled alpha/gamma oscillations, showing similar contrast sensitivities for both.

After 8 weeks of PATH training, large improvements in working memory (VWM improved from $34 \%$ to $86 \%$, AWM improved from 55\% to $97 \%$ ) were found for a healthy 71 years-old adult who was already adept at paying attention, yet still improved after training (81\% to $87 \%)$, see Table 2 . She also improved substantially in processing speed: from $42 \%$ to $77 \%$. Her reading skills improved markedly, more than doubling in her reading speed (229 to 541 words/min), dyslexia improving from markedly or mildly below normal to above normal, and Wide Range Achievement Tests (WRAT) of Reading, Spelling and Math improved, Math increasing from $73 \%$ to 95\%, as shown in Table 2. These results show that not only reading, attention, processing speed and working memory skills, but also problem solving skills improved markedly. These results show cognitive transfer to untrained tasks. Since coupled alpha-gamma activity is reduced in older adults with mild cognitive impairments [68], these improvements in working memory provide more evidence PATH training improves coupled alpha-gamma activity 
Table 2. Older Adult Pre-Post Standardized Percentiles and Reading Scores

\begin{tabular}{|l|l|l|}
\hline Standardized Tests & Pre-Test & Post-Test \\
\hline Adult Dyslexia Test (ADT) Visual Processing & Markedly Below Normal & Above Normal \\
\hline ADT Phonological Processing & Mildly Below Normal & Above Normal \\
\hline WRAT Reading & $75 \%$ & $87 \%$ \\
\hline WRAT Spelling & $58 \%$ & $61 \%$ \\
\hline WRAT Math & $73 \%$ & $95 \%$ \\
\hline Reading Speed & 229 words/min & 541 words/min \\
\hline WAIS Processing Speed & $42 \%$ & $77 \%$ \\
\hline DKEFS Attention & $81 \%$ & $87 \%$ \\
\hline DKEFS Cognitive Flexibility & $81 \%$ & $87 \%$ \\
\hline TIPS Visual Working Memory & $34 \%$ & $86 \%$ \\
\hline WAIS Auditory Working Memory & $55 \%$ & $97 \%$ \\
\hline
\end{tabular}

Following PATH neurotraining, this older adult's quality of life improved remarkably. She reported "My memory, reading speed, ease of understanding, processing speed, ability to multitask, concentrate, and pay attention have improved remarkably in just a few months. Since doing PATH, I find that driving is much easier, and I am able to attend to a much wider region, allowing me to see street signs more easily. I am now able to distinguish the other car movements at dawn and dusk much better improving my driving skills. These improvements in remembering, concentrating, and reading have made life much easier and more enjoyable. I have noticed that everyday activities are so much easier to complete and are more enjoyable. I hope that you are able to help other older adults so that forgetting and everyday activities are no longer difficult."

These results demonstrate neuroplasticity in the domain of visual processing, processing speed, reading speed, attention, working memory, and problem solving using massed practice discriminating moving patterns that optimally activate magnocells. We found, using MEG source imaging, that speeding up these motion cells improves the attention and executive control networks of both young adults who are dyslexic, and older adults with mild cognitive impairments. Previously, PATH training was shown to improve visual and cognitive skills in dyslexic and typically developing children [1-3,6], older adults [62], and an adolescent and adults 50-68 years old after a concussion from a mTBI [5]. We will now demonstrate that these timing deficits are prevalent in the dorsal stream of dyslexics, older adults, and those with a mTBI.

\section{Discussion}

\subsection{Theory of Change}

The scientific premise for using PATH training is that remediation of a fundamental visual timing deficit affecting motion discrimination at a low level of cognitive processing generalizes to high level cognitive skills (attention, working memory, processing speed, and reading) reliant upon motion processing as a foundation. We propose that by improving the slow processing speed of magnocells at low levels of the dorsal stream (V1MT), we are improving subsequently higher levels in the dorsal stream (PPC) improving visual and auditory attention, and dlPFC improving working memory, cognitive flexibility, and problem solving, and subsequently reading speed and comprehension. This movement discrimination training enhances coupled theta/gamma and alpha/gamma oscillations, improving both the feedforward and feedback attention pathways used by the dIPFC to modulate attention in MT and V1, enabling a wide range of cognitive skills to improve. This theory of change, see Fig. 2, has been validated by the results of the MEG 
imaging study below and the improvements in cognitive skills following only 8 weeks of 15-20 min twice a week of PATH training, as described below.

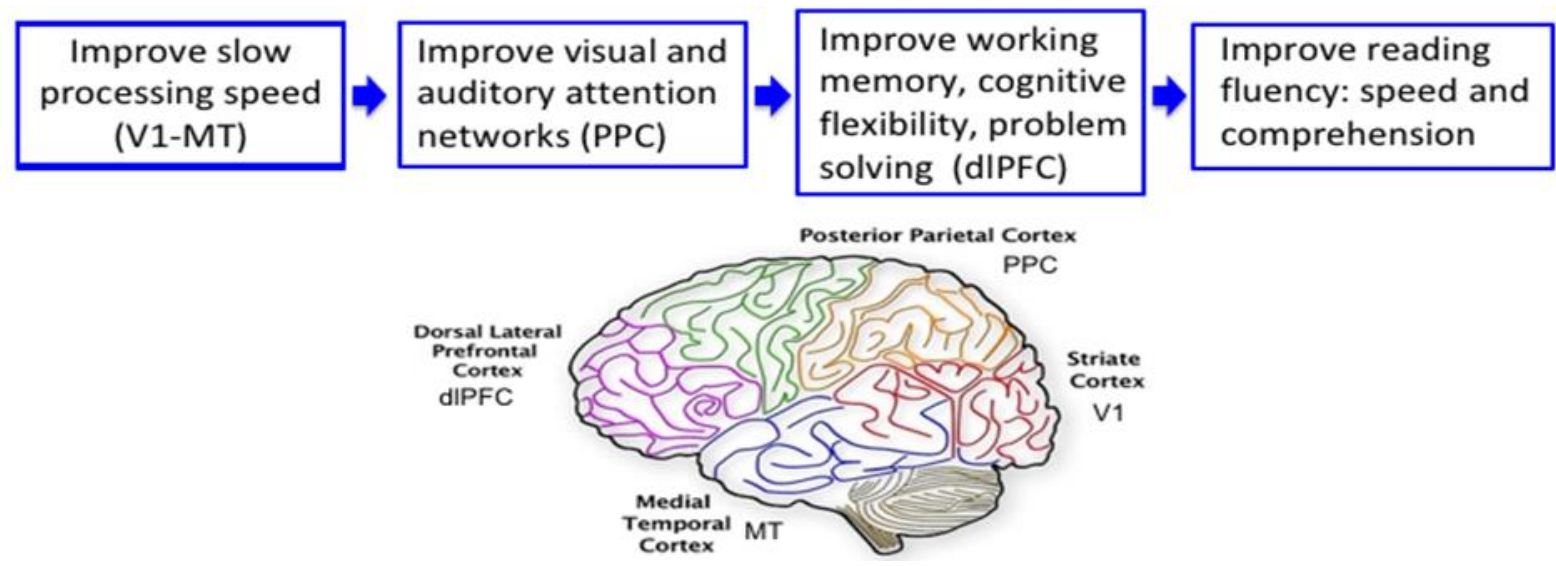

Figure 2. Theory of Change proposes that low-level timing deficits (V1-MT) improve processing at subsequently higher cortical levels of processing, being the primary factor causing impairments in cortical processing for developmental disorders, aging, and concussions.

\subsection{Timing Deficits Prevalent in Dyslexics Remediated by Movement Discrimination Exercises}

Dyslexics have magnocellular responses that were found to be $20-40 \mathrm{~ms}$ slower than typically developing observers $[55,56]$, being $2-4$ fold slower than the normal magnocellular lead time of 10-20 ms [94,95]. Some investigators hypothesize that in dyslexics a lack of synchronization in timing between magnocellular and parvocellular activations may prevent effective sequential processing, pattern analysis, and figure/ground discrimination, and hence impede the development of efficient reading and attention skills $[1,2,3,6,7,8,13,14,16,29,30,57,66,93,96,97]$. Our working hypothesis $[3,7]$ is that magnocellular neurons in the dorsal cortical visual pathway (V1-MT) of dyslexics are sluggish, causing visual timing deficits at lower levels of visual processing [55,56]. These disrupt processing at higher levels of dorsal stream processing, as shown by dyslexics having little or no activity in MT $[98,99]$, including the development of these visual and attention pathways. These visual timing deficits limit reading acquisition in dyslexics.

Convergent evidence finds that many dyslexic readers demonstrate impairments in movement discrimination tasks that rely upon magnocellular functioning. Dyslexics were found to have motion perception deficits at each of these levels of processing in the dorsal stream:

1. the retinal level when measured using the frequency doubling illusion [100-103],

2. the Lateral Geniculate Nucleus (LGN) where the magnocellular layers were found to be $30 \%$ smaller and more disorganized [55],

3. V1 measured using VEPs [55, 56, 104,105],

4. V1 and MT using both fMRI brain imaging $[98,99]$ and MEG brain imaging [4], and psychophysical tasks of movement discrimination relative to a stationary background $[1-3,7,13,96]$,

5. MT using motion coherence for direction discrimination [106-111],

6. the Lateral IntraParietal cortex (LIP) and Frontal Eye Fields (FEF), anterior cortical areas activated by saccades, based on saccade and anti-saccade training tasks [61], causing text to appear to move, a symptom that many dyslexics report [112,113], and

7. parietal structures, prefrontal language systems, cerebellum, basal ganglia [114], and hubs of the attention networks $[4,5]$.

These results suggest a strong relationship between dorsal stream processing and reading ability, such that poor dorsal stream processing caused by sluggish magno (motion) cells is associated with slower timing and poor reading skills $[1,2,3,13,14,16,40,57,66,93,96,97,102,107,115-117]$. In fact, motion sensitivity in individuals 
predicts orthographic reading skills in good and poor readers [118,119]. Dyslexics have sluggish motion cells that do not properly signal the pattern-sensitive cells, causing difficulty in isolating and identifying the critical elements needed for reading, such as the beginning and end of the word before sequentially analyzing the letters in the word. In fact, research finds that there is an imbalance in magno- and parvo-cellular systems in dyslexics [120]. Dyslexics thereby lack the ability to process sequential information quickly and accurately, causing deficits in both reading speed and comprehension.

Just by doing rapid brain exercises that improve a person's ability to discriminate left-right movement relative to a stationary background pattern, PATH neurotraining, significantly improves a person's ability to read rapidly and accurately. These brain exercises can be used to not only detect slow reading, dyslexia and other developmental problems, like speech and language, attention deficits, autism, schizophrenia, and concussions earlier than other methods, but also they can be used to remediate the cognitive deficits rapidly and effectively, as shown extensively in controlled validation studies in schools and with individual patients $[1-3,6,13]$ by improving the brain's timing as shown in this study and previously $[4,5,105]$.

Since only in MT and higher processing levels are neurons selectively sensitive to motion coherence [121,122], direction discrimination using motion coherence has not been found to be an effective training paradigm [123]. Instead vertical sinewave gratings are used for PATH training, since these activate all motion sensitive neurons throughout all visual cortical areas. Studies that have questioned the hypothesis that dyslexics have magnocellular deficits [124-127] examined dyslexics' sensitivity to stimuli that are not optimal for activating direction-selective cells in the V1-MT network [71,128], using either flicker or high contrast random dot patterns without a background pattern.

The PATH movement-discrimination training patterns, vertical sinewave gratings, are designed to differentially activate motion-sensitive (magnocellular) neurons in the V1MT network [20,32,71,129,130] relative to pattern-sensitive (parvocellular) neurons, thereby being an effective training stimulus to improve magno-parvo integration, and timing deficits at both early and higher levels of visual motion processing. Contrast sensitivity for Figure (moving test stripes) / Ground (stationary background stripes) discrimination is needed to provide an effective training stimulus to improve dorsal stream function [1-3,6,7]. The MEG results presented in Fig. 3 from an adult dyslexic corroborates these findings, showing that the timing and sensitivity of magnocells in MT improve significantly after a short amount of PATH training. Moreover, finding P1, N1, and P2, N2 MT signals improve markedly after only 5 hours of training on movement discrimination indicates that attentional signals driven by both alpha/gamma and theta/gamma oscillations, respectively, are enhanced [87]. The results from this study suggest that a short amount of movement discrimination training enhances coupled alpha/gamma and theta/gamma oscillations, improving attentional modulation, and the cognitive skills of reading, attention, processing speed, and working memory in dyslexics, as found previously [3,6]. Furthermore, there is evidence that improvements in the cognitive skills of dyslexics after this movement-discrimination training that is more rapid and effective than the competition are sustained over time [2].

\subsection{Timing Deficits Prevalent in Older Adults Remediated by Movement Discrimination Exercises}

Older adults have deficits in visual processing [131], motion discrimination [132], attention [133], and working memory [134], timing deficits being a prevalent factor. Agebased changes in task-switching also is likely due to a slowing of perceptual processing [135]. Results from behavioral methodologies have indicated a general age-related decline in cognitive functions such as speed of processing, attention, perception, and working memory [133-135] that have been verified by fMRI and PET scans as well [136-139]. The data from this study provide new evidence that the deficits in attentional focus, working memory, and navigation experienced by older adults result from an information overload 
due to timing deficits in the direction-selectivity network that are abated following training on movement discrimination using a dynamic visual attention (PATH) therapy. These results support a previous study [62] suggesting that magnocellular pathways provide the gateway for attentive processing for older adults.

Lawton and Stephey's [62] data suggest that training on movement discrimination, designed to strengthen magnocellular activity, increased the ability of the dorsal pathway to improve navigation and memory. These studies provide evidence that in older adults the timing in their brain is disrupted, affecting attention, processing speed, memory, multitasking, and navigation. Slower processing speeds and more effortful attention have been found to explain a large part of age-related memory deterioration $[133,135,138]$. Reduced information processing speed may explain problems in memory encoding and retrieval because this mental slowing can lead to superficial processing and inefficient strategies where elaboration is required [140].

Although a general slowing of information processing [135], as well as declines in bottom-up sensory processes [131,132] may account for many age-related changes, deficits in selective attention do appear to exist in several domains: feature selection, object-based attention, and temporal attention [133]. Furthermore, age-related declines in spatial search appear to be attributable to deficits in selective attention, yet it is unclear whether this reflects deficient top-down mechanisms or insufficient compensation to overcome bottom-up declines [133]. Since this study found that improving bottom-up timing improved high-level cognitive skills requiring coupled theta/gamma and alpha/gamma oscillations, this indicates bottom-up processing is the limiting factor in cognitive skills declining as we age. This conclusion is supported by the neural plasticity underlying visual perceptual learning in aging following training on a movement discrimination task designed to activate MST [87]. This study showed that following training on slow (theta) and fast (alpha) speeds of movement, improvements in the ERP were found in the first $300 \mathrm{msec}$, as also found in the MEG recordings in Fig. 3, improving the allocation of attention. The perceptual training of older adults by Mishra et al. [87] produced large improvements in speed and accuracy, but no improvements in cognitive skills validated by neuropsychological tests, like those shown in Table 2, were reported. It is likely PATH training, activating magnocells at both early and late levels of dorsal stream processing is more effective in improving attention, processing speed, and memory in older adults.

It may be that aging causes a decline in frontal inhibitory control over posterior brain regions [141], thereby having reduced coupled theta/gamma and alpha/gamma oscillations. This hypothesis is supported by fMRI data that establishes that top-down modulation mediated by the PFC, subserved by alpha band activity, provides a causal link between early attentional processes and subsequent memory performance [142]. Adults with cognitive deficits / dementia may have decreases in the proper PFC areas within the working memory (WM) network, but over recruitment from other PFC regions outside the proper WM network [136,139]. Since this study found that a short amount of movement discrimination training improved an older adult's reading, attention, processing speed, problem solving, and both visual and auditory working memory skills, this data provides irrefutable evidence that improving low-level dorsal stream activity by increasing the timing and sensitivity of magnocells enhances coupled theta/gamma and alpha/gamma oscillations that enable improving high-level cognitive functions. It is likely that after PATH training improves the timing and sensitivity in the dorsal stream, older adults have enhanced activity in the proper WM network, and reduced activity from regions outside the WM network, as found in the dyslexic adult in this study, and following a mTBI [5].

\subsection{Timing deficits Prevalent After a Concussion Caused by mTBI Remediated by Movement Discrimination Exercises}

Research has found that the effects of a mTBI reflect disruptions of the neural networks for attention and working memory [143-146]. After a mTBI, patients have trouble in sustaining attention [147]. Cognitive deficits in those with a mTBI are hypothesized to 
result from neural timing deficits [148]. Compensation for timing issues by increased prefrontal cortical recruitment would be manifest as increased distractibility, working memory deficits, and problems with balance and coordination. This expended effort may underlie fatigue, headache, irritability, anxiety, and when prolonged, depression [148]. Visual timing deficits, resulting from magnocellular (motion) deficits often persist after a mild TBI [149], manifesting as timing deficits in the dorsal pathways, and attention and executive control networks [5,148]. Thus, daily living is dramatically impaired after a mTBI.

The magnocellular deficits persistent in those having a mTBI [149] were remediated quickly [5] by completing a short amount of movement-discrimination (PATH) training designed to optimally activate magnocellular (left-right movement) relative to parvocellular (stationary background) neurons in the dorsal stream. After a mTBI in people aged 15-68 years, substantial MEG signal increases in Motion Networks (V1, V3, MT, MST) and Attention / Memory Networks (ACC, precuneous/PCC, vlPFC, and dlPFC areas) were found following PATH training 2 times/week for 8-16 weeks, as well as significant behavioral improvements in movement discrimination, processing speed, reading speed, both sustained and selective attention, and both auditory and visual working memory [5]. These are the same improvements found in this study for the dyslexic adult. The data suggest that the PATH movement-discrimination training paradigm improves magnocellular function, which improves processing speed, attention, memory, and reading, in part by improving attentional feedback to V1 and MT, that can be measured by the strength of coupled theta/gamma and alpha/gamma frequency oscillations [15]. Our data found that PATH exercises improved processing speed, attention, working memory, problem solving, and reading, so less effort had to be spent decoding information, hence more effort was available for interpreting the information, improving timing by using the working memory network more efficiently [26], skills that improve a person's quality of life after a mTBI. PATH training in mTBI patients [5] is the first time that improving low-level visual timing deficits in the dorsal stream were found to improve high-level cognitive functioning, both behaviorally and using a biomarker, MEG physiological brain recordings.

Other cognitive training programs: 1) had little effect on improving the executive functions and attention in mTBI $[150,151]$, 2) had results from brain training that were neither robust nor consistent, with transfer and sustained effects which were limited [152], and 3) improved the task being trained on, but do not extend to tasks not trained on or everyday cognitive performance [74]. Currently, there are no proven solutions to improve attention and working memory in $\mathrm{mTBI}$ patients $[64,150,153,154]$. However, not only attention and memory, but also reading and processing speed improved significantly in mTBI patients after a short amount of movement discrimination exercises [5].

\section{Conclusions}

What emerges from multiple studies is the essential role of timing deficits in the dorsal stream that are prevalent in developmental disorders like dyslexia, in aging, and following a mTBI. Training visual dorsal stream function at low levels (V1-MT pathways) significantly improved high-level cognitive functions, including reading fluency, selective and sustained attention, processing speed, problem solving, and working memory. This study provides irrefutable evidence that these improvements in cognitive skills result from increasing the temporal precision and neuronal sensitivity of magnocellular neurons in MT relative to linked parvocellular neurons in the dorsal stream. PATH movementdiscrimination training was faster and more effective in improving reading, attention, and memory for dyslexics than found after training on: 1) repeated reading interventions, 2) interventions designed to improve auditory timing, or 3) linguistic-based reading interventions. This training was found to be rapid and greatly accelerate learning, improving reading, attention, processing speed, and memory more than found previously, since it: 1) improved neural timing by improving the function of magnocells using figure/ground movement-discrimination at both low and high levels in the dorsal stream, improving 
feedforward and feedback pathways to modulate attention by enhancing coupled theta/gamma and alpha/gamma oscillations, 2) is adaptive on a trial-by-trial basis based on the participant's performance, and 3) incorporated cycles of feedback and reward at multiple levels. Remediating visual timing deficits in the dorsal stream revealed the causal role of visual movement discrimination training to facilitate reading acquisition in dyslexic and typically-developing students between the ages of 6 to 8 years old. Moreover, this research supports the hypothesis that faulty timing in synchronizing the activity of magnocellular with parvocellular visual pathways in the dorsal stream is a fundamental cause of dyslexia and argues that the phonological reading deficiencies in dyslexia are caused by impaired development of the visual magnocellular timing system. These studies indicate that a paradigm shift in treating dyslexia from phonologically-based to visually-based methods is called for urgently. In addition, a paradigm shift in treating reading, attention, processing speed, and memory impairments in older adults, and after a concussion is needed as well. Improving the timing and sensitivity of low-level dorsal pathways, improving feedforward and feedback pathways to modulate attention, is essential to improve high-level cognitive skills: reading, attention, processing speed, working memory, and problem solving in dyslexics, older adults, and those with a mTBI quickly and effectively.

Author Contributions: Conceptualization, T.L., J.S.-T., M.-X.H.; Methodology, T.L., M.-X.H.; Software, T.L., M.-X.H.; Validation, T.L., J.S.-T., M.-X.H.; Formal Analysis, T.L., J.S.-T., M.-X.H.; Investigation, T.L., M.-X.H.; Writing - Original Draft Preparation, T.L. M.-X.H.; Writing - Review \& Editing, T.L., J.S.-T., M.-X.H.; Supervision, T.L., M.-X.H.

Funding: This research received no external funding.

Institutional Review Board Statement: In this section, please add the Institutional Review Board Statement and approval number for studies involving humans or animals. Please note that the Editorial Office might ask you for further information. Please add "The study was conducted according to the guidelines of the Declaration of Helsinki, and approved by the Institutional Review Board (or Ethics Committee) of NAME OF INSTITUTE (protocol code XXX and date of approval)." OR "Ethical review and approval were waived for this study, due to REASON (please provide a detailed justification)." OR "Not applicable." for studies not involving humans or animals. You might also choose to exclude this statement if the study did not involve humans or animals.

Informed Consent Statement: Informed consent was obtained from all subjects involved in the study.

Data Availability Statement: The data is not publically archived at this time. Interested parties should contact the authors.

Acknowledgements. We thank Dr. John Stein for his insightful revisions on a previous version of this paper.

Conflicts of Interest: Authors two and three declare no conflict of interest. Only the first author has any competing interests. Although she developed the PATH neurotraining that is now available as a web-app, she had no influence over the data that was collected, so could not influence the results in any way.

\section{References}

1. Lawton, T. Training Direction-Discrimination Sensitivity Remediates a Wide Spectrum of Reading Skills. Optom Vis Develop 2007, 38, 37-51.

2. Lawton, T. Improving Magnocellular Function in the Dorsal Stream Remediates Reading Deficits. Optom Vis Develop 2011, 42, 142-154.

3. Lawton, T. Improving Dorsal Stream Function in Dyslexics by Training Figure/Ground Motion Discrimination Improves Attention, Reading Fluency, and Working Memory. Front Hum Neurosci 2016, 397, doi:10.3389/fnhum.2016.00397. 
4. Lawton, T.; Huang, M.X. Improving Visual Timing Enhances Cognitive Functioning by Altering Dorsal Stream and Attention Networks.; 12th Annual World Congress of the Society for Brain Mapping and Therapeutics, Los Angeles, CA, 2015.

5. Lawton, T.; Huang, M.-X. Dynamic Cognitive Remediation for a Traumatic Brain Injury (TBI) Significantly Improves Attention, Working Memory, Processing Speed, and Reading Fluency. Restor Neurol Neurosci 2019, 37, 71-86, doi:10.3233/RNN-180856.

6. Lawton, T.; Shelley-Tremblay, J. Training on Movement Figure-Ground Discrimination Remediates Low-Level Visual Timing Deficits in the Dorsal Stream, Improving High-Level Cognitive Functioning, Including Attention, Reading Fluency, and Working Memory. Front Hum Neurosci 2017, 11, doi:10.3389/fnhum.2017.00236.

7. Lawton, T.A. Methods and Apparatus for Diagnosing and Remediating Reading Disorders 2000. US Patent No. $6,045,515,6,213956$ B1.

8. Lawton, T. Diagnosing and Remediating Cognitive Deficits Involving Attention, Sequential Processing, Reading, Speed of Processing, and Navigation. 2015. US Patent No. 8,979,263B2. https://www.freepatentsonline.com/8979263.html.

9. Buzsaki, G. Rhythms of the Brain; Oxford University Press, 2006.

10. Lawton, T. The Effect of Phase Structures on Spatial Phase Discrimination. Vision Res 1984, 24, 139-148.

11. Lawton, T.B. Spatial-Frequency Spectrum of Patterns Changes the Visibility of Spatial-Phase Differences. J. Opt. Soc. Am. A, JOSA A 1985, 2, 1140-1152, doi:10.1364/JOSAA.2.001140.

12. Lawton, T. Outputs of Paired Gabor Filters Summed across the Background Frame of Reference Predict the Direction of Movement (Vision). IEEE Trans Biomed Eng 1989, 36, 130-139.

13. Lawton, T. Filtered Text and Direction Discrimination Training Improved Reading Fluency for Both Dyslexic and Normal Readers. Optom Vis Develop 2008, 39, 114-126.

14. Lawton, T. Training Directionally Selective Motion Pathways Can Significantly Improve Reading Efficiency.; $2004 ;$ Vol. 5292, pp. 34-45.

15. Vidyasagar, T.R. Reading into Neuronal Oscillations in the Visual System: Implications for Developmental Dyslexia Front Hum Neurosci 2013, 7, doi:10.3389/fnhum.2013.00811.

16. Vidyasagar, T.R. A Neuronal Model of Attentional Spotlight: Parietal Guiding the Temporal. Brain Res Rev 1999, 30 , 66-76, doi:10.1016/S0165-0173(99)00005-3.

17. Livingstone, M.; Hubel, D. Segregation of Form, Color, Movement, and Depth: Anatomy, Physiology, and Perception. Science $1988,240,740-749$.

18. Maunsell, J.H.R.; Nealey, T.A.; DePriest, D.D. Magnocellular and Parvocellular Contributions to Responses in the Middle Temporal Visual Area (MT) of the Macaque Monkey. J Neurosc 1990, 10, 3323-3334.

19. Merigan, W.H.; Maunsell, J.H.R. How Parallel Are the Primate Visual Pathways? Ann Rev Neurosci 1993, 16, 369-402.

20. Felleman, D.J.; Van Essen, D.C. Distributed Hierarchical Processing in the Primate Cerebral Cortex. Cereb Cortex 1991, $1,1-47$.

21. Ungerleider, L.G.; Mishkin, M. Two cortical visual systems. In Analysis of Visual Behavior.; Ingle, D.J., Goodale, M.A., Mansfield, R.J.W., Eds.; Elsevier, 1982; Vol. 6, pp. 549-586.

22. Kolster, H.; Peeters, R.; Orban, G. The Human MT/V5 Cluster. J Neurosci 2010, 30, 9801-9820.

23. Pitzalis, S.; Sereno, M.I.; Committeri, G.; Fattori, P.; Galati, G.; Patria, F.; Galletti, C. Human V6: The Medial Motion Area. Cereb Cortex 2010, 20, 411-424, doi:10.1093/cercor/bhp112.

24. Posner, M.I.; Walker, J.A.; Friedrich, F.J.; Rafal, R.D. Effects of Parietal Injury on Covert Orienting of Attention. J Neurosci 1984,4 , 1863-1874.

25. Battelli, L.; Pascual-Leone, A.; Cavanagh, P. The 'When' Pathway of the Right Parietal Lobe. TiCS 2007, 11, 204-210, doi:10.1016/j.tics.2007.03.001.

26. Menon, V.; Uddin, L.Q. Saliency, Switching, Attention and Control: A Network Model of Insula Function. Brain Struct Funct 2010, 214, 655-667, doi:10.1007/s00429-010-0262-0.

27. Titz, C.; Karbach, J. Working Memory and Executive Functions: Effects of Training on Academic Achievement. Psychol Res 2014, 78, 852-868, doi:10.1007/s00426-013-0537-1. 
28. Brain Futures, Brain Fitness and Executive Function: Evidence-Based Interventions That Improve Student Outcomes. Available online: www.brainfutures.org, 2019.

29. Vidyasagar, T.R. From Attentional Gating in Macaque Primary Visual Cortex to Dyslexia in Humans. Prog Brain Res 2001, 134, 297-312.

30. Vidyasagar, T.R. Visual Aspects of Dyslexia. In Visual Aspects of Dyslexia; Stein, J., Kapoula, Z., Eds.; Oxford University Press, 2012; pp. 151-170 ISBN 978-0-19-958981-4.

31. Vidyasagar, T.R. Visual Attention and Neural Oscillations in Reading and Dyslexia: Are They Possible Targets for Remediation? Neuropsychologia 2019, 130, 59-65, doi:10.1016/j.neuropsychologia.2019.02.009.

32. Hupé, J.M.; James, A.C.; Payne, B.R.; Lomber, S.G.; Girard, P.; Bullier, J. Cortical Feedback Improves Discrimination between Figure and Background by V1, V2 and V3 Neurons. Nature 1998, 394, 784-787, doi:10.1038/29537.

33. Kaplan, E.; Shapley, R.M. The Primate Retina Contains Two Types of Ganglion Cells, with High and Low Contrast Sensitivity. PNAS 1986, 83, 2755-2757.

34. Sclar, G.; Maunsell, J.H.R.; Lennie, P. Coding of Image Contrast in Central Visual Pathways of the Macaque Monkey. Vision Res 1990, 30, 1-10, doi:10.1016/0042-6989(90)90123-3.

35. Silver, M.A.; Ress, D.; Heeger, D.J. Topographic Maps of Visual Spatial Attention in Human Parietal Cortex. J Neurophysiol 2005, 94, 1358-1371, doi:10.1152/jn.01316.2004.

36. Watanabe, T.; Sasaki, Y.; Miyauchi, S.; Putz, B.; Fujimaki, N.; Nielsen, M.; Takino, R.; Miyakawa, S. Attention-Regulated Activity in Human Primary Visual Cortex. J Neurophysiol 1998, 79, 2218-2221, doi:10.1152/jn.1998.79.4.2218.

37. Martínez, A.; Anllo-Vento, L.; Sereno, M.I.; Frank, L.R.; Buxton, R.B.; Dubowitz, D.J.; Wong, E.C.; Hinrichs, H.; Heinze H.J.; Hillyard, S.A. Involvement of Striate and Extrastriate Visual Cortical Areas in Spatial Attention. Nat Neurosci 1999, 2, 364-369, doi:10.1038/7274.

38. Somers, D.C.; Dale, A.M.; Seiffert, A.E.; Tootell, R.B.H. Functional MRI Reveals Spatially Specific Attentional Modulation in Human Primary Visual Cortex. PNAS 1999, 96, 1663-1668, doi:10.1073/pnas.96.4.1663.

39. Buschman, T.J.; Miller, E.K. Top-down versus Bottom-up Control of Attention in the Prefrontal and Posterior Parietal Cortices. Science 2007, 315, 1860-1862.

40. Saalmann, Y.B.; Pigarev, I.N.; Vidyasagar, T.R. Neural Mechanisms of Visual Attention: How Top-Down Feedback Highlights Relevant Locations. Science 2007, 316, 1612-1615, doi:10.1126/science.1139140.

41. Brefczynski, J.A.; DeYoe, E.A. A Physiological Correlate of the'spotlight'of Visual Attention. Nat Neurosci 1999, 2, 370374.

42. Archer, K.; Pammer, K.; Vidyasagar, T.R. A Temporal Sampling Basis for Visual Processing in Developmental Dyslexia. Front Hum Neurosci 2020, 14, doi:10.3389/fnhum.2020.00213.

43. Saalmann, Y.B.; Pinsk, M.A.; Wang, L.; Li, X.; Kastner, S. The Pulvinar Regulates Information Transmission Between Cortical Areas Based on Attention Demands. Science 2012, 337, 753-756, doi:10.1126/science.1223082.

44. Dehaene, S. Reading in the Brain 9780143118053 । PenguinRandomHouse.Com: Books; Viking: Penguin Group: New York, NY, US, 2009.

45. Pammer, K.; Hansen, P.; Holliday, I.; Cornelissen, P. Attentional Shifting and the Role of the Dorsal Pathway in Visual Word Recognition. Neuropsychologia 2006, 44, 2926-2936, doi:10.1016/j.neuropsychologia.2006.06.028.

46. Facoetti, A.; Zorzi, M.; Cestnick, L.; Lorusso, M.L.; Molteni, M.; Paganoni, P.; Umiltà, C.; Mascetti, G.G. The Relationship between Visuo-Spatial Attention and Nonword Reading in Developmental Dyslexia. Cogn Neuropsychol 2006, 23, 841855

47. Solan, H.A.; Larson, S.; Shelley-Tremblay, J.; Ficarra, A.; Silverman, M. Role of Visual Attention in Cognitive Control of Oculomotor Readiness in Students with Reading Disabilities. J Learn Disabil 2001, 34, 107-118, doi:10.1177/002221940103400202.

48. Vidyasagar, T.R.; Pammer, K. Dyslexia: A Deficit in Visuo-Spatial Attention, Not in Phonological Processing. TiCS 2010, 14, 57-63, doi:10.1016/j.tics.2009.12.003

49. Stuart, G.W.; McAnally, K.I.; Castles, A. Can Contrast Sensitivity Functions in Dyslexia Be Explained by Inattention Rather than a Magnocellular Deficit? Vision Res 2001, 41, 3205-3211, doi:10.1016/S0042-6989(01)00176-6.

50. Hyafil, A.; Fontolan, L.; Kabdebon, C.; Gutkin, B.; Giraud, A.-L. Speech Encoding by Coupled Cortical Theta and Gamma Oscillations. eLife 2015, 4, e06213, doi:10.7554/eLife.06213. 
51. Goswami, U. A Temporal Sampling Framework for Developmental Dyslexia. TiCS 2011, 15, 3-10, doi:10.1016/j.tics.2010.10.001

52. Vidyasagar, T.R.; Levichkina, E. An Integrated Neuronal Model of Claustral Function in Timing the Synchrony Between Cortical Areas. Front Neural Circuits 2019, 13, doi:10.3389/fncir.2019.00003.

53. Hyafil, A.; Giraud, A.-L.; Fontolan, L.; Gutkin, B. Neural Cross-Frequency Coupling: Connecting Architectures, Mechanisms, and Functions. Trends Neurosci 2015, 38, 725-740, doi:10.1016/j.tins.2015.09.001

54. Canolty, R.T.; Knight, R.T. The Functional Role of Cross-Frequency Coupling. TiCS 2010, 14, 506-515.

55. Livingstone, M.; Rosen, G.D.; Drislane, F.W.; Galaburda, A.M. Physiological and Anatomical Evidence for a Magnocellular Defect in Developmental Dyslexia. PNAS 1991, 88, 7943-7947.

56. Lehmkuhle, S.; Garzia, R.P.; Turner, L.; Hash, T.; Baro, J.A. A Defective Visual Pathway in Children with Reading Disability. NEJM 1993, 328, 989-996, doi:10.1056/NEJM199304083281402.

57. Lawton, T. Increasing Visual Timing by Movement Discrimination Exercises Improves Reading Fluency, Attention Span, and Memory Retention in Dyslexics. Neurol Neurosurg 2019, 2, 1-8.

58. O'Leary, J.G.; Lisberger, S.G. Role of the Lateral Intraparietal Area in Modulation of the Strength of Sensory-Motor Transmission for Visually Guided Movements. J Neurosci 2012, 32, 9745-9754, doi:10.1523/JNEUROSCI.0269-12.2012.

59. Slaghuis, W.L.; Ryan, J.F. Spatio-Temporal Contrast Sensitivity, Coherent Motion, and Visible Persistence in Developmental Dyslexia. Vision Res 1999, 39, 651-668, doi:10.1016/S0042-6989(98)00151-5.

60. Slaghuis, W.L.; Ryan, J.F. Directional Motion Contrast Sensitivity in Developmental Dyslexia. Vision Res 2006, 46, 32913303, doi:10.1016/j.visres.2006.05.009.

61. Fischer, B. Subitizing, dynamic vision, saccade and fixation control in dyslexia. In Visual Aspects of Dyslexia; Stein, J., Kapoula, Z., Eds.; OUP Oxford, 2012; pp. 15-43 ISBN 978-0-19-958981-4.

62. Lawton, T.; Stephey, D. Field of View, Figure/Ground Discrimination, Sequential Memory, and Navigation Skills Improve Following Training on Motion Discrimination in Older Adults. Optom Vis Develop 2009, 40, 82-93.

63. Wise, B.W.; Ring, J.; Olson, R.K. Individual Differences in Gains from Computer-Assisted Remedial Reading. J Exp Child Psychol 2000, 77, 197-235, doi:10.1006/jecp.1999.2559.

64. Raymer, A.M.; Roitsch, J.; Redman, R.; Michalek, A.M.P.; Johnson, R.K. Critical Appraisal of Systematic Reviews of Executive Function Treatments in TBI. Brain Injury 2018, 32, 1601-1611, doi:10.1080/02699052.2018.1522671.

65. Brown, A.C.; Peters, J.L.; Parsons, C.; Crewther, D.P.; Crewther, S.G. Efficiency in Magnocellular Processing: A Common Deficit in Neurodevelopmental Disorders. Front Hum Neurosci 2020, 14, doi:10.3389/fnhum.2020.00049.

66. Stein, J. The Current Status of the Magnocellular Theory of Developmental Dyslexia. Neuropsychologia 2019, 130, 6677, doi:10.1016/j.neuropsychologia.2018.03.022.

67. Albright, T.D. Direction and Orientation Selectivity of Neurons in Visual Area MT of the Macaque. J Neurophysiol 1984, 52, 1106-1130, doi:10.1152/jn.1984.52.6.1106.

68. Chen, Y.; He, H.; Xu, P.; Wang, J.; Qiu, Y.; Feng, W.; Luo, Y.; Hu, L.; Guan, Q. The Weakened Relationship Between Prestimulus Alpha Oscillations and Response Time in Older Adults With Mild Cognitive Impairment. Front Hum Neurosci 2020, 14, doi:10.3389/fnhum.2020.00048.

69. Blakemore, C.; Campbell, F.W. On the Existence of Neurones in the Human Visual System Selectively Sensitive to the Orientation and Size of Retinal Images. J Physiol 1969, 203, 237-260.

70. De Valois, R.L.; Albrecht, D.G.; Thorell, L.G. Spatial Frequency Selectivity of Cells in Macaque Visual Cortex. Vision Res 1982, 22, 545-559.

71. De Valois, R.L.; Cottaris, N.P.; Mahon, L.E.; Elfar, S.D.; Wilson, J.A. Spatial and Temporal Receptive Fields of Geniculate and Cortical Cells and Directional Selectivity. Vision Res 2000, 40, 3685-3702.

72. Higgins, K.E.; Jaffe, M.J.; Coletta, N.J.; Caruso, R.C.; de Monasterio, F.M. Spatial Contrast Sensitivity: Importance of Controlling the Patient's Visibility Criterion. Arch Ophthalmol 1984, 102, 1035-1041.

73. Dobres, J.; Watanabe, T. Response Feedback Triggers Long-Term Consolidation of Perceptual Learning Independently of Performance Gains. J Vision 2012, 12, 1-10. 
74. Simons, D.J.; Boot, W.R.; Charness, N.; Gathercole, S.E.; Chabris, C.F.; Hambrick, D.Z.; Stine-Morrow, E.A. Do “BrainTraining" Programs Work? Psychological Science in the Public Interest 2016, 17, 103-186.

75. Owen, A.M.; McMillan, K.M.; Laird, A.R.; Bullmore, E. N-Back Working Memory Paradigm: A Meta-Analysis of Normative Functional Neuroimaging Studies. Hum Brain Mapp 2005, 25, 46-59.

76. Phillips, N.L.; Parry, L.; Mandalis, A.; Lah, S. Working Memory Outcomes Following Traumatic Brain Injury in Children: A Systematic Review with Meta-Analysis. Child Neuropsychol 2017, 23, 26-66, doi:10.1080/09297049.2015.1085500.

77. Taulu, S.; Kajola, M.; Simola, J. Suppression of Interference and Artifacts by the Signal Space Separation Method. Brain Topogr 2004, 16, 269-275, doi:10.1023/B:BRAT.0000032864.93890.f9.

78. Taulu, S.; Simola, J.; Kajola, M. MEG Recordings of DC Fields Using the Signal Space Separation Method (SSS). Neurol Clin Neurophysiol: NCN 2004, 35, 1-4

79. Song, T.; Gaa, K.; Cui, L.; Feffer, L.; Lee, R.R.; Huang, M. Evaluation of Signal Space Separation via Simulation. Med Biol Eng Comput 2008, 46, 923-932, doi:10.1007/s11517-007-0290-y.

80. Hyvarinen, A. Fast and Robust Fixed-Point Algorithms for Independent Component Analysis. IEEE Transactions on Neural Networks 1999, 10, 626-634, doi:10.1109/72.761722.

81. Hyvärinen, A.; Oja, E. Independent Component Analysis: Algorithms and Applications. Neural Networks 2000, 13, 411-430, doi:10.1016/S0893-6080(00)00026-5.

82. Huang, M.-X.; Huang, C.W.; Robb, A.; Angeles, A.; Nichols, S.L.; Baker, D.G.; Song, T.; Harrington, D.L.; Theilmann R.J.; Srinivasan, R.; et al. MEG Source Imaging Method Using Fast L1 Minimum-Norm and Its Applications to Signals with Brain Noise and Human Resting-State Source Amplitude Images. NeuroImage 2014, 84, 585-604, doi:10.1016/j.neuroimage.2013.09.022.

83. Huang, C.W.; Huang, M.-X.; Ji, Z.; Swan, A.R.; Angeles, A.M.; Song, T.; Huang, J.W.; Lee, R.R. High-Resolution MEG Source Imaging Approach to Accurately Localize Broca's Area in Patients with Brain Tumor or Epilepsy. Clin Neurophysiol 2016, 127, 2308-2316, doi:10.1016/j.clinph.2016.02.007.

84. Grabner, G.; Janke, A.L.; Budge, M.M.; Smith, D.; Pruessner, J.; Collins, D.L. Symmetric Atlasing and Model Based Segmentation: An Application to the Hippocampus in Older Adults. In: Proceedings of the Medical Image Computing and Computer-Assisted Intervention - MICCAI 2006; Larsen, R., Nielsen, M., Sporring, J., Eds.; Springer: Berlin, Heidelberg, 2006; pp. 58-66.

85. Smith, S.M.; Jenkinson, M.; Woolrich, M.W.; Beckmann, C.F.; Behrens, T.E.J.; Johansen-Berg, H.; Bannister, P.R.; De Luca, M.; Drobnjak, I.; Flitney, D.E.; et al. Advances in Functional and Structural MR Image Analysis and Implementation as FSL. NeuroImage 2004, 23, S208-S219, doi:10.1016/j.neuroimage.2004.07.051.

86. Woolrich, M.W.; Jbabdi, S.; Patenaude, B.; Chappell, M.; Makni, S.; Behrens, T.; Beckmann, C.; Jenkinson, M.; Smith, S.M. Bayesian Analysis of Neuroimaging Data in FSL. NeuroImage 2009, 45, S173-S186, doi:10.1016/j.neuroimage.2008.10.055

87. Mishra, J.; Rolle, C.; Gazzaley, A. Neural Plasticity Underlying Visual Perceptual Learning in Aging. Brain Res 2015, 1612, 140-151, doi:10.1016/j.brainres.2014.09.009.

88. Hillyard, S.A.; Anllo-Vento, L. Event-Related Brain Potentials in the Study of Visual Selective Attention. PNAS 1998, 95, 781-787, doi:10.1073/pnas.95.3.781.

89. Hillyard, S.A.; Vogel, E.K.; Luck, S.J. Sensory Gain Control (Amplification) as a Mechanism of Selective Attention Electrophysiological and Neuroimaging Evidence. Philos Trans R Soc Lond B Biol Sci 1998, 353, 1257-1270, doi:10.1098/rstb.1998.0281.

90. Richlan, F. Developmental Dyslexia: Dysfunction of a Left Hemisphere Reading Network. Front Hum Neurosci 2012, 6, doi:10.3389/fnhum.2012.00120.

91. Vadasy, P.F.; Sanders, E.A. Repeated Reading Intervention: Outcomes and Interactions with Readers' Skills and Classroom Instruction. J Educ Psychol 2008, 100, 272-290, doi:10.1037/0022-0663.100.2.272.

92. Thatcher, R.W.; Walker, R.A.; Giudice, S. Human Cerebral Hemispheres Develop at Different Rates and Ages. Science 1987, 236, 1110-1113

93. Stein, J. The Magnocellular Theory of Developmental Dyslexia. Dyslexia 2001, 7, 12-36, doi:10.1002/dys.186

94. Dreher, B.; Fukada, Y.; Rodieck, R.W. Identification, Classification and Anatomical Segregation of Cells with X-like and Y-like Properties in the Lateral Geniculate Nucleus of Old-World Primates. J Physiol 1976, 258, 433-452, doi:https://doi.org/10.1113/jphysiol.1976.sp011429. 
95. Nowak, L.G.; Munk, M.H.J.; Girard, P.; Bullier, J. Visual Latencies in Areas V1 and V2 of the Macaque Monkey. Visual Neurosci 1995, 12, 371-384, doi:10.1017/S095252380000804X.

96. Lawton, T. Training Direction Selectivity Significantly Improves Reading Fluency for All Types of Inefficient Readers. J Vision 2004, 4, 765 .

97. Stein, J.; Walsh, V. To See but Not to Read; the Magnocellular Theory of Dyslexia. Trends Neurosci 1997, 20, 147-152, doi:10.1016/S0166-2236(96)01005-3.

98. Demb, J.B.; Boynton, G.M.; Heeger, D.J. Functional Magnetic Resonance Imaging of Early Visual Pathways in Dyslexia. J Neurosci 1998, 18, 6939-6951.

99. Eden, G.F.; VanMeter, J.W.; Rumsey, J.M.; Maisog, J.M.; Woods, R.P.; Zeffiro, T.A. Abnormal Processing of Visual Motion in Dyslexia Revealed by Functional Brain Imaging. Nature 1996, 382, 66-69, doi:10.1038/382066a0.

100. Avellis, F.; Darsò, A.; Gandolfi, S.; Carta, A. Magnocellular Deficit in Dyslexia: A Preliminary Analysis of Possible Patterns of Visual Field Deficit Tested with Frequency Doubling Illusion. Transl Biomed 2016, 7:4 doi: 10.2167/2172-0479.100094.

101. Buchholz, J.; McKone, E. Adults with Dyslexia Show Deficits on Spatial Frequency Doubling and Visual Attention Tasks. Dyslexia 2004, 10, 24-43.

102. Gori, S.; Cecchini, P.; Bigoni, A.; Molteni, M.; Facoetti, A. Magnocellular-Dorsal Pathway and Sub-Lexical Route in Developmental Dyslexia. Front Hum Neurosci 2014, 8, 460, doi:10.3389/fnhum.2014.00460.

103. Kevan, A.; Pammer, K. Predicting Early Reading Skills from Pre-Reading Measures of Dorsal Stream Functioning. Neuropsychologia 2009, 47, 3174-3181, doi:10.1016/j.neuropsychologia.2009.07.016.

104. Crewther, S.G.; Crewther, D.P. Development of the Magnocellular VEP in Children: Implications for Reading Disability. Electroencephalography and Clinical Neurophysiology 1999, Suppl. 49, 123-128.

105. Shelley-Tremblay, J.; Syklawer, S.; Ramkissoon, I. The Effects of Magno-Parvocellular Integration Training on Fluency and Visual Evoked Potentials in Poor Readers. J Behav Optom 2011, 222, 31-38.

106. Boets, B.; Vandermosten, M.; Cornelissen, P.; Wouters, J.; Ghesquière, P. Coherent Motion Sensitivity and Reading Development in the Transition from Prereading to Reading Stage. Child Develop 2011, 82, 854-869.

107. Gori, S.; Seitz, A.R.; Ronconi, L.; Franceschini, S.; Facoetti, A. Multiple Causal Links Between Magnocellular-Dorsal Pathway Deficit and Developmental Dyslexia. Cereb Cortex 2016, 26, 4356-4369, doi:10.1093/cercor/bhv206.

108. Hansen, P.C.; Stein, J.F.; Orde, S.R.; Winter, J.L.; Talcott, J.B. Are Dyslexics' Visual Deficits Limited to Measures of Dorsal Stream Function? NeuroReport 2001, 12, 1527-1530.

109. Talcott, J.B.; Hansen, P.C.; Willis-Owen, C.; McKinnell, I.W.; Richardson, A.J.; Stein, J.F. Visual Magnocellular Impairment in Adult Developmental Dyslexics. Neuro-Ophthalmol 1998, 20, 187-201, doi:10.1076/noph.20.4.187.3931.

110. Talcott, J.B.; Hansen, P.C.; Assoku, E.L.; Stein, J.F. Visual Motion Sensitivity in Dyslexia: Evidence for Temporal and Energy Integration Deficits. Neuropsychologia 2000, 38, 935-943, doi:10.1016/S0028-3932(00)00020-8.

111. Cornelissen, P.; Richardson, A.; Mason, A.; Fowler, S.; Stein, J. Contrast Sensitivity and Coherent Motion Detection Mea sured at Photopic Luminance Levels in Dyslexics and Controls. Vision Res 1995, 35, 1483-1494, doi:10.1016/0042-6989(95)98728R.

112. Singleton, C.; Trotter, S. Visual Stress in Adults with and without Dyslexia. J Res Read 2005, 28, 365-378, doi:https://doi.org/10.1111/j.1467-9817.2005.00275.x.

113. Harries, P.; Hall, R.; Ray, N.; Stein, J.F. Using Coloured Filters to Reduce the Symptoms of Visual Stress in Children with Reading Delay. Scand J Occup Ther 2015, 22, 153-160, doi:https://doi.org/10.3109/11038128.2014.989903.

114. Nicolson, R.I.; Fawcett, A.J. Procedural Learning Difficulties: Reuniting the Developmental Disorders? Trends Neurosci 2007, 30, 135-141, doi:10.1016/j.tins.2007.02.003.

115. Stein, J. The Current Status of the Magnocellular Theory of Developmental Dyslexia. Neuropsychologia 2018, 130, 66-77. doi:10.1016/j.neuropsychologia.2018.03.022.

116. Stein, J.F. Does Dyslexia Exist? Lang Cogn Neurosci 2018, 33, 313-320, doi:10.1080/23273798.2017.1325509.

117. Vidyasagar, T.R. Chapter 19 From attentional gating in macaque primary visual cortex to dyslexia in humans. In Progress in Brain Research; Vision: From Neurons to Cognition; Elsevier, 2001; Vol. 134, pp. 297-312. 
118. Witton, C.; Talcott, J.B.; Hansen, P.C.; Richardson, A.J.; Griffiths, T.D.; Rees, A.; Stein, J.F.; Green, G.G. Sensitivity to Dynamic Auditory and Visual Stimuli Predicts Nonword Reading Ability in Both Dyslexic and Normal Readers. Curr Biol 1998, $8,791-797$.

119. Talcott, J.B.; Witton, C.; Hebb, G.S.; Stoodley, C.J.; Westwood, E.A.; France, S.J.; Hansen, P.C.; Stein, J.F. On the Relationship between Dynamic Visual and Auditory Processing and Literacy Skills; Results from a Large Primary-School Study. Dyslexia 2002, 8, 204-225, doi:10.1002/dys.224.

120. Ciavarelli, A.; Contemori, G.; Battaglini, L.; Barollo, M.; Casco, C. Dyslexia and the Magnocellular-Parvocellular Coactivaton Hypothesis. Vision Res 2021, 179, 64-74, doi:10.1016/j.visres.2020.10.008.

121. Braddick, O.J.; O’Brien, J.M.D.; Wattam-Bell, J.; Atkinson, J.; Hartley, T.; Turner, R. Brain Areas Sensitive to Coherent Visual Motion. Perception 2001, 30, 61-72, doi:10.1068/p3048.

122. Zohary, E.; Celebrini, S.; Britten, K.H.; Newsome, W.T. Neuronal Plasticity That Underlies Improvement in Perceptual Performance. Science 1994, 263, 1289-1292.

123. Solan, H.A.; Shelley-Tremblay, J.; Hansen, P.C.; Silverman, M.E.; Larsone, S.; Ficarra, A. M-Cell Deficit and Reading Disability: A Preliminary Study of the Effects of Temporal Vision-Processing Therapy. Optometry-J Am Optom Assoc 2004, 75, 640-650.

124. Amitay, S.; Ben - Yehudah, G.; Banai, K.; Ahissar, M. Disabled Readers Suffer from Visual and Auditory Impairments but Not from a Specific Magnocellular Deficit. Brain 2002, 125, 2272 - 2285, doi:10.1093/brain/awf231.

125. Skottun, B.C. The Magnocellular Deficit Theory of Dyslexia: The Evidence from Contrast Sensitivity. Vision Res 2000, 40 , 111-127, doi:10.1016/S0042-6989(99)00170-4.

126. Skottun, B.C.; Skoyles, J.R. Coherent Motion, Magnocellular Sensitivity and the Causation of Dyslexia. International J Neurosci 2008, 118, 185-190, doi:10.1080/00207450601041872.

127. Williams, M.J.; Stuart, G.W.; Castles, A.; McAnally, K.I. Contrast Sensitivity in Subgroups of Developmental Dyslexia. Vision Res 2003, 43, 467-477, doi:10.1016/S0042-6989(02)00573-4.

128. Baker, C.L. Spatial and Temporal Determinants of Directionally Selective Velocity Preference in Cat Striate Cortex Neurons. J Neurophysiol 1988, 59, 1557-1574.

129. Allman, J.; Miezin, F.; McGuinness, E. Stimulus Specific Responses from beyond the Classical Receptive Field: Neurophysiological Mechanisms for Local-Global Comparisons in Visual Neurons. Ann Rev Neurosci 1985, 8, 407-430.

130. Nassi, J.J.; Lyon, D.C.; Callaway, E.M. The Parvocellular LGN Provides a Robust Disynaptic Input to the Visual Motion Area MT. Neuron 2006, 50, 319-327, doi:10.1016/j.neuron.2006.03.019.

131. Owsley, C. Aging and Vision. Vision Res 2011, 51, 1610-1622, doi:10.1016/j.visres.2010.10.020.

132. Bennett, P.J.; Sekuler, R.; Sekuler, A.B. The Effects of Aging on Motion Detection and Direction Identification. Vision Res 2007, 47, 799-809, doi:10.1016/j.visres.2007.01.001.

133. Zanto, T.P.; Gazzaley, A. Attention and ageing. In The Oxford handbook of attention; Oxford library of psychology; Oxford University Press: New York, NY, US, 2014; pp. 927-971 ISBN 978-0-19-967511-1.

134. Craik, F.I. Memory Changes in Normal Aging. Curr Dir Psychol Sci 1994, 3, 155-158.

135. Salthouse, T.A. Aging and Measures of Processing Speed. Biol Psychol 2000, 54, 35-54, doi:10.1016/S0301-0511(00)00052-1.

136. Grady, C.L.; Maisog, J.M.; Horwitz, B.; Ungerleider, L.G.; Mentis, M.J.; Salerno, J.A.; Pietrini, P.; Wagner, E.; Haxby, J.V. Age-Related Changes in Cortical Blood Flow Activation during Visual Processing of Faces and Location. J Neurosci 1994, 14, 1450-1462, doi:10.1523/JNEUROSCI.14-03-01450.1994.

137. Cabeza, R.; Kapur, S.; Craik, F.I.M.; McIntosh, A.R.; Houle, S.; Tulving, E. Functional Neuroanatomy of Recall and Recognition: A PET Study of Episodic Memory. J Cogn Neurosci 1997, 9, 254-265, doi:10.1162/jocn.1997.9.2.254.

138. Tisserand, D.J.; Jolles, J. On the Involvement of Prefrontal Networks in Cognitive Ageing. Cortex 2003, 39, 1107-1128, doi:10.1016/S0010-9452(08)70880-3.

139. Dennis, N.A.; Kim, H.; Cabeza, R. Age-Related Differences in Brain Activity during True and False Memory Retrieval. J Cogn Neurosci 2008, 20, 1390-1402, doi:10.1162/jocn.2008.20096.

140. Jolles, J. Chapter 2 Cognitive, emotional and behavioral dysfunctions in aging and dementia. In Progress in Brain Research; Swaab, D.F., Fliers, E., Mirmiran, M., Van Gool, W.A., Van Haaren, F., Eds.; Aging of the Brain and Alzheimer's Disease; Elsevier, 1986; Vol. 70, pp. 15-39. 
141. Goldman-Rakic, P.S. Topography of Cognition: Parallel Distributed Networks in Primate Association Cortex. Ann Rev Neurosci 1988, 11, 137-156, doi:10.1146/annurev.ne.11.030188.001033.

142. Zanto, T.P.; Rubens, M.T.; Thangavel, A.; Gazzaley, A. Causal Role of the Prefrontal Cortex in Top-down Modulation of Visual Processing and Working Memory. Nat Neurosci 2011, 14, 656-661, doi:10.1038/nn.2773.

143. Huang, M.-X.; Nichols, S.; Robb-Swan, A.; Angeles-Quinto, A.; Harrington, D.L.; Drake, A.; Huang, C.W.; Song, T.; Diwakar, M.; Risbrough, V.B.; et al. MEG Working Memory N-Back Task Reveals Functional Deficits in Combat-Related Mild Traumatic Brain Injury. Cereb Cortex 2019, 29, 1953-1968, doi:10.1093/cercor/bhy075.

144. Knight, R.T. Neural Networks Debunk Phrenology. Science 2007, 316, 1578-1579.

145. Kennedy, M.R.; Coelho, C.; Turkstra, L.; Ylvisaker, M.; Moore Sohlberg, M.; Yorkston, K.; Chiou, H.-H.; Kan, P.-F. Intervention for Executive Functions after Traumatic Brain Injury: A Systematic Review, Meta-Analysis and Clinical Recommendations. Neuropsychol Rehabil 2008, 18, 257-299.

146. Dikmen, S.S.; Corrigan, J.D.; Levin, H.S.; Machamer, J.; Stiers, W.; Weisskopf, M.G. Cognitive Outcome Following Traumatic Brain Injury. J Head Trauma Rehabil 2009, 24, 430-438, doi:10.1097/HTR.0b013e3181c133e9.

147. Huisman, T.A.G.M.; Schwamm, L.H.; Schaefer, P.W.; Koroshetz, W.J.; Shetty-Alva, N.; Ozsunar, Y.; Wu, O.; Sorensen, A.G. Diffusion Tensor Imaging as Potential Biomarker of White Matter Injury in Diffuse Axonal Injury. Am J Neuroradiol 2004, 25, 370-376.

148. Ghajar, J.; Ivry, R.B. The Predictive Brain State: Timing Deficiency in Traumatic Brain Injury? Neurorehabil Neural Repair $2008,22,217-227$.

149. Poltavski, D.; Lederer, P.; Cox, L.K. Visually Evoked Potential Markers of Concussion History in Patients with Convergence Insufficiency. Optom Vis Sci 2017, 94, 742-750.

150. Hallock, H.; Collins, D.; Lampit, A.; Deol, K.; Fleming, J.; Valenzuela, M. Cognitive Training for Post-Acute Traumatic Brain Injury: A Systematic Review and Meta-Analysis. Front Hum Neurosci 2016, 10, doi:10.3389/fnhum.2016.00537.

151. Bogdanova, Y.; Yee, M.K.; Ho, V.T.; Cicerone, K.D. Computerized Cognitive Rehabilitation of Attention and Executive Function in Acquired Brain Injury: A Systematic Review. J Head Trauma Rehabil 2016, 31, 419-433.

152. Buitenweg, J.I.V.; Murre, J.M.J.; Ridderinkhof, K.R. Brain Training in Progress: A Review of Trainability in Healthy Seniors. Front Hum Neurosci 2012, 6, doi:10.3389/fnhum.2012.00183.

153. Rohling, M.L.; Faust, M.E.; Beverly, B.; Demakis, G. Effectiveness of Cognitive Rehabilitation Following Acquired Brain Injury: A Meta-Analytic Re-Examination of Cicerone et al.'s (2000, 2005) Systematic Reviews. Neuropsychology 2009, 23, 2039, doi:10.1037/a0013659.

154. Roitsch, J.; Redman, R.; Michalek, A.M.P.; Johnson, R.K.; Raymer, A.M. Quality Appraisal of Systematic Reviews for Behavioral Treatments of Attention Disorders in Traumatic Brain Injury. J Head Trauma Rehabil 2019, 34, E42-E50, doi:10.1097/HTR.0000000000000444. 